Supporting Information

\title{
Tapping on the Black Box: How is the Scoring Power of a Machine-Learning Scoring Function Dependent on the Training Set?
}

Minyi Su, ${ }^{1,2}$ Guoqin Feng, ${ }^{1,2}$ Zhihai Liu, ${ }^{1}$ Yan Li, ${ }^{1,3}$ and Renxiao Wang $1,3,4$ *

${ }^{1}$ State Key Laboratory of Bioorganic and Natural Products Chemistry, Center for Excellence in Molecular Synthesis, Shanghai Institute of Organic Chemistry, Chinese Academy of Sciences, 345 Lingling Road, Shanghai 200032, People's Republic of China

${ }^{2}$ University of Chinese Academy of Sciences, Beijing 100049, People's Republic of China

${ }^{3}$ Department of Medicinal Chemistry, School of Pharmacy, Fudan University, 826 Zhangheng Road, Shanghai 201203, People's Republic of China

${ }^{4}$ Shanxi Key Laboratory of Innovative Drugs for the Treatment of Serious Diseases Basing on Chronic Inflammation, College of Traditional Chinese Medicines, Shanxi University of Chinese Medicine, Taiyuan, Shanxi 030619, People's Republic of China

*: To whom all correspondence should be addressed. E-mail: wangrx@fudan.edu.cn 


\section{TABLE OF CONTENTS}

PART 1. Performance of all models calibrated on the training sets with different levels of similarity by protein sequence 3

PART 2. Performance of all models calibrated on the training sets with different levels of similarity by ligand shape

PART 3. Performance of all models calibrated on the training sets with different levels of similarity by binding pocket

PART 4. Performance of all models calibrated on the training sets with different levels of similarity by the 3-in-

1 combined metric

PART 5. Performance of all models calibrated on the training sets with different sizes (average similarity defined by protein sequence)

PART 6. Performance of all models calibrated on the training sets with different sizes (average similarity defined by ligand shape)

PART 7. Performance of all models calibrated on the training sets with different sizes (average similarity defined by binding pocket)

PART 8. Performance of all models calibrated on the training sets with different sizes (average similarity defined by the 3 -in- 1 combined metric)

PART 9. List of the non-redundant, standard training sets compiled based on the PDBbind refined set 22

PART 10. Performance of all scoring functions calibrated on the non-redundant training sets selected from several versions of the PDBbind refined set 
PART 1. Performance of all models calibrated on the training sets with different levels of similarity by protein sequence

Table S1. Pearson correlation coefficients produced by all models on the CASF-2016 test set

\begin{tabular}{|c|c|c|c|c|c|c|c|c|c|}
\hline $\begin{array}{l}\text { Similarity } \\
\text { to test set }\end{array}$ & X-Score ${ }^{\mathrm{HM}}$ & ChemScore & ASP & L-SVR & RF & KNN & DT & BRR & MLP \\
\hline 0.92 & $0.628 \pm 0.003$ & $0.584 \pm 0.003$ & $0.489 \pm 0.000$ & $0.666 \pm 0.006$ & $0.805 \pm 0.004$ & $0.670 \pm 0.002$ & $0.657 \pm 0.012$ & $0.680 \pm 0.002$ & $0.537 \pm 0.078$ \\
\hline 0.90 & $0.629 \pm 0.003$ & $0.585 \pm 0.004$ & $0.489 \pm 0.000$ & $0.665 \pm 0.008$ & $0.803 \pm 0.004$ & $0.674 \pm 0.003$ & $0.666 \pm 0.012$ & $0.684 \pm 0.002$ & $0.534 \pm 0.081$ \\
\hline 0.88 & $0.635 \pm 0.003$ & $0.587 \pm 0.004$ & $0.489 \pm 0.000$ & $0.668 \pm 0.007$ & $0.797 \pm 0.006$ & $0.675 \pm 0.004$ & $0.660 \pm 0.013$ & $0.685 \pm 0.003$ & $0.554 \pm 0.087$ \\
\hline 0.86 & $0.633 \pm 0.004$ & $0.591 \pm 0.005$ & $0.487 \pm 0.001$ & $0.649 \pm 0.009$ & $0.785 \pm 0.007$ & $0.674 \pm 0.004$ & $0.646 \pm 0.012$ & $0.679 \pm 0.004$ & $0.536 \pm 0.087$ \\
\hline 0.84 & $0.632 \pm 0.004$ & $0.595 \pm 0.005$ & $0.486 \pm 0.001$ & $0.657 \pm 0.017$ & $0.778 \pm 0.008$ & $0.675 \pm 0.005$ & $0.637 \pm 0.015$ & $0.674 \pm 0.005$ & $0.527 \pm 0.096$ \\
\hline 0.82 & $0.630 \pm 0.005$ & $0.596 \pm 0.005$ & $0.484 \pm 0.002$ & $0.633 \pm 0.030$ & $0.779 \pm 0.008$ & $0.675 \pm 0.005$ & $0.640 \pm 0.014$ & $0.670 \pm 0.005$ & $0.542 \pm 0.079$ \\
\hline 0.80 & $0.626 \pm 0.005$ & $0.597 \pm 0.005$ & $0.482 \pm 0.002$ & $0.640 \pm 0.021$ & $0.776 \pm 0.009$ & $0.675 \pm 0.005$ & $0.640 \pm 0.016$ & $0.667 \pm 0.005$ & $0.545 \pm 0.083$ \\
\hline 0.78 & $0.624 \pm 0.006$ & $0.598 \pm 0.006$ & $0.481 \pm 0.003$ & $0.635 \pm 0.017$ & $0.771 \pm 0.010$ & $0.674 \pm 0.005$ & $0.632 \pm 0.016$ & $0.665 \pm 0.006$ & $0.531 \pm 0.094$ \\
\hline 0.76 & $0.625 \pm 0.005$ & $0.599 \pm 0.006$ & $0.480 \pm 0.003$ & $0.642 \pm 0.016$ & $0.763 \pm 0.012$ & $0.675 \pm 0.006$ & $0.625 \pm 0.018$ & $0.663 \pm 0.006$ & $0.541 \pm 0.096$ \\
\hline 0.74 & $0.626 \pm 0.005$ & $0.604 \pm 0.005$ & $0.479 \pm 0.004$ & $0.645 \pm 0.014$ & $0.760 \pm 0.010$ & $0.671 \pm 0.006$ & $0.619 \pm 0.017$ & $0.664 \pm 0.006$ & $0.527 \pm 0.090$ \\
\hline 0.72 & $0.627 \pm 0.006$ & $0.603 \pm 0.006$ & $0.478 \pm 0.005$ & $0.648 \pm 0.016$ & $0.758 \pm 0.013$ & $0.673 \pm 0.006$ & $0.615 \pm 0.019$ & $0.666 \pm 0.007$ & $0.530 \pm 0.095$ \\
\hline 0.70 & $0.628 \pm 0.006$ & $0.603 \pm 0.006$ & $0.477 \pm 0.005$ & $0.649 \pm 0.018$ & $0.748 \pm 0.014$ & $0.673 \pm 0.007$ & $0.605 \pm 0.021$ & $0.667 \pm 0.007$ & $0.535 \pm 0.086$ \\
\hline 0.68 & $0.630 \pm 0.005$ & $0.607 \pm 0.006$ & $0.478 \pm 0.005$ & $0.637 \pm 0.021$ & $0.742 \pm 0.015$ & $0.676 \pm 0.007$ & $0.595 \pm 0.024$ & $0.660 \pm 0.007$ & $0.508 \pm 0.101$ \\
\hline 0.66 & $0.628 \pm 0.004$ & $0.608 \pm 0.006$ & $0.477 \pm 0.005$ & $0.636 \pm 0.023$ & $0.726 \pm 0.015$ & $0.675 \pm 0.006$ & $0.579 \pm 0.022$ & $0.656 \pm 0.009$ & $0.529 \pm 0.091$ \\
\hline 0.64 & $0.627 \pm 0.005$ & $0.608 \pm 0.006$ & $0.476 \pm 0.005$ & $0.635 \pm 0.021$ & $0.715 \pm 0.014$ & $0.677 \pm 0.005$ & $0.575 \pm 0.021$ & $0.659 \pm 0.008$ & $0.520 \pm 0.085$ \\
\hline 0.62 & $0.631 \pm 0.004$ & $0.617 \pm 0.004$ & $0.479 \pm 0.004$ & $0.628 \pm 0.026$ & $0.718 \pm 0.012$ & $0.678 \pm 0.006$ & $0.577 \pm 0.020$ & $0.658 \pm 0.008$ & $0.494 \pm 0.102$ \\
\hline 0.60 & $0.630 \pm 0.004$ & $0.616 \pm 0.005$ & $0.480 \pm 0.004$ & $0.626 \pm 0.024$ & $0.704 \pm 0.014$ & $0.679 \pm 0.006$ & $0.565 \pm 0.022$ & $0.655 \pm 0.009$ & $0.507 \pm 0.093$ \\
\hline 0.58 & $0.629 \pm 0.005$ & $0.617 \pm 0.004$ & $0.480 \pm 0.004$ & $0.626 \pm 0.025$ & $0.694 \pm 0.014$ & $0.678 \pm 0.006$ & $0.557 \pm 0.024$ & $0.654 \pm 0.009$ & $0.497 \pm 0.106$ \\
\hline 0.56 & $0.629 \pm 0.004$ & $0.618 \pm 0.004$ & $0.480 \pm 0.003$ & $0.625 \pm 0.022$ & $0.690 \pm 0.015$ & $0.680 \pm 0.005$ & $0.557 \pm 0.019$ & $0.654 \pm 0.009$ & $0.502 \pm 0.086$ \\
\hline 0.54 & $0.628 \pm 0.004$ & $0.618 \pm 0.004$ & $0.480 \pm 0.003$ & $0.626 \pm 0.024$ & $0.693 \pm 0.011$ & $0.675 \pm 0.006$ & $0.555 \pm 0.020$ & $0.665 \pm 0.007$ & $0.500 \pm 0.103$ \\
\hline 0.52 & $0.626 \pm 0.004$ & $0.620 \pm 0.004$ & $0.479 \pm 0.003$ & $0.612 \pm 0.027$ & $0.672 \pm 0.013$ & $0.670 \pm 0.006$ & $0.532 \pm 0.023$ & $0.657 \pm 0.008$ & $0.515 \pm 0.087$ \\
\hline
\end{tabular}


Table S2. RMSE produced by all models on the CASF-2016 test set

\begin{tabular}{|c|c|c|c|c|c|c|c|c|c|}
\hline $\begin{array}{l}\text { Similarity } \\
\text { to test set }\end{array}$ & $\mathrm{X}$-Score ${ }^{\mathrm{HM}}$ & ChemScore & ASP & L-SVR & RF & KNN & DT & BRR & MLP \\
\hline 0.92 & $1.79 \pm 0.01$ & $1.80 \pm 0.00$ & $1.90 \pm 0.00$ & $2.33 \pm 0.05$ & $1.36 \pm 0.01$ & $1.67 \pm 0.01$ & $1.64 \pm 0.02$ & $1.63 \pm 0.00$ & $3.69 \pm 0.81$ \\
\hline 0.90 & $1.78 \pm 0.01$ & $1.79 \pm 0.01$ & $1.90 \pm 0.00$ & $2.35 \pm 0.05$ & $1.36 \pm 0.01$ & $1.66 \pm 0.01$ & $1.63 \pm 0.02$ & $1.62 \pm 0.00$ & $3.59 \pm 0.84$ \\
\hline 0.88 & $1.76 \pm 0.01$ & $1.78 \pm 0.01$ & $1.90 \pm 0.00$ & $2.30 \pm 0.06$ & $1.38 \pm 0.01$ & $1.65 \pm 0.01$ & $1.64 \pm 0.03$ & $1.62 \pm 0.01$ & $3.54 \pm 0.91$ \\
\hline 0.86 & $1.76 \pm 0.01$ & $1.78 \pm 0.01$ & $1.90 \pm 0.00$ & $2.27 \pm 0.07$ & $1.41 \pm 0.01$ & $1.66 \pm 0.01$ & $1.66 \pm 0.02$ & $1.64 \pm 0.01$ & $3.68 \pm 0.94$ \\
\hline 0.84 & $1.76 \pm 0.01$ & $1.78 \pm 0.01$ & $1.90 \pm 0.00$ & $2.20 \pm 0.09$ & $1.42 \pm 0.02$ & $1.66 \pm 0.01$ & $1.68 \pm 0.03$ & $1.65 \pm 0.01$ & $3.76 \pm 0.93$ \\
\hline 0.82 & $1.75 \pm 0.01$ & $1.77 \pm 0.01$ & $1.90 \pm 0.00$ & $2.28 \pm 0.12$ & $1.42 \pm 0.02$ & $1.65 \pm 0.01$ & $1.67 \pm 0.03$ & $1.65 \pm 0.01$ & $3.79 \pm 1.10$ \\
\hline 0.80 & $1.75 \pm 0.01$ & $1.77 \pm 0.01$ & $1.91 \pm 0.00$ & $2.27 \pm 0.11$ & $1.43 \pm 0.02$ & $1.65 \pm 0.01$ & $1.67 \pm 0.03$ & $1.66 \pm 0.01$ & $3.64 \pm 0.80$ \\
\hline 0.78 & $1.75 \pm 0.01$ & $1.77 \pm 0.01$ & $1.91 \pm 0.01$ & $2.30 \pm 0.12$ & $1.45 \pm 0.02$ & $1.65 \pm 0.01$ & $1.69 \pm 0.03$ & $1.67 \pm 0.01$ & $3.76 \pm 1.01$ \\
\hline 0.76 & $1.75 \pm 0.01$ & $1.77 \pm 0.01$ & $1.92 \pm 0.01$ & $2.27 \pm 0.13$ & $1.46 \pm 0.03$ & $1.65 \pm 0.01$ & $1.70 \pm 0.03$ & $1.67 \pm 0.01$ & $3.78 \pm 0.95$ \\
\hline 0.74 & $1.75 \pm 0.01$ & $1.76 \pm 0.01$ & $1.92 \pm 0.01$ & $2.22 \pm 0.11$ & $1.47 \pm 0.02$ & $1.66 \pm 0.01$ & $1.71 \pm 0.03$ & $1.68 \pm 0.01$ & $3.83 \pm 0.84$ \\
\hline 0.72 & $1.75 \pm 0.01$ & $1.77 \pm 0.01$ & $1.93 \pm 0.01$ & $2.20 \pm 0.12$ & $1.48 \pm 0.03$ & $1.67 \pm 0.01$ & $1.72 \pm 0.03$ & $1.68 \pm 0.01$ & $3.81 \pm 0.95$ \\
\hline 0.70 & $1.75 \pm 0.01$ & $1.77 \pm 0.01$ & $1.94 \pm 0.01$ & $2.18 \pm 0.10$ & $1.50 \pm 0.03$ & $1.67 \pm 0.01$ & $1.73 \pm 0.04$ & $1.68 \pm 0.01$ & $3.81 \pm 0.83$ \\
\hline 0.68 & $1.75 \pm 0.01$ & $1.77 \pm 0.01$ & $1.94 \pm 0.01$ & $2.24 \pm 0.12$ & $1.52 \pm 0.03$ & $1.67 \pm 0.01$ & $1.75 \pm 0.04$ & $1.70 \pm 0.01$ & $4.04 \pm 0.99$ \\
\hline 0.66 & $1.75 \pm 0.01$ & $1.77 \pm 0.01$ & $1.95 \pm 0.01$ & $2.25 \pm 0.14$ & $1.55 \pm 0.03$ & $1.67 \pm 0.01$ & $1.78 \pm 0.04$ & $1.71 \pm 0.01$ & $3.92 \pm 0.96$ \\
\hline 0.64 & $1.75 \pm 0.01$ & $1.77 \pm 0.01$ & $1.96 \pm 0.01$ & $2.25 \pm 0.13$ & $1.57 \pm 0.03$ & $1.67 \pm 0.01$ & $1.78 \pm 0.03$ & $1.71 \pm 0.01$ & $4.00 \pm 0.93$ \\
\hline 0.62 & $1.74 \pm 0.01$ & $1.76 \pm 0.01$ & $1.96 \pm 0.01$ & $2.28 \pm 0.13$ & $1.56 \pm 0.02$ & $1.66 \pm 0.01$ & $1.78 \pm 0.04$ & $1.72 \pm 0.01$ & $4.21 \pm 1.02$ \\
\hline 0.60 & $1.74 \pm 0.01$ & $1.76 \pm 0.01$ & $1.97 \pm 0.01$ & $2.26 \pm 0.12$ & $1.59 \pm 0.02$ & $1.66 \pm 0.01$ & $1.80 \pm 0.04$ & $1.72 \pm 0.01$ & $3.99 \pm 0.95$ \\
\hline 0.58 & $1.75 \pm 0.01$ & $1.76 \pm 0.01$ & $1.98 \pm 0.01$ & $2.27 \pm 0.12$ & $1.60 \pm 0.02$ & $1.67 \pm 0.01$ & $1.82 \pm 0.04$ & $1.73 \pm 0.01$ & $4.13 \pm 0.99$ \\
\hline 0.56 & $1.75 \pm 0.01$ & $1.76 \pm 0.01$ & $1.99 \pm 0.02$ & $2.28 \pm 0.11$ & $1.61 \pm 0.03$ & $1.67 \pm 0.01$ & $1.82 \pm 0.03$ & $1.73 \pm 0.01$ & $3.99 \pm 0.83$ \\
\hline 0.54 & $1.76 \pm 0.01$ & $1.77 \pm 0.01$ & $1.99 \pm 0.02$ & $2.26 \pm 0.11$ & $1.61 \pm 0.02$ & $1.69 \pm 0.01$ & $1.82 \pm 0.03$ & $1.74 \pm 0.01$ & $4.19 \pm 1.09$ \\
\hline 0.52 & $1.77 \pm 0.01$ & $1.78 \pm 0.01$ & $2.01 \pm 0.02$ & $2.30 \pm 0.13$ & $1.65 \pm 0.02$ & $1.70 \pm 0.01$ & $1.86 \pm 0.04$ & $1.76 \pm 0.01$ & $3.97 \pm 0.94$ \\
\hline
\end{tabular}


Table S3. Spearman correlation coefficients produced by all models on the CASF-2016 test set

\begin{tabular}{|c|c|c|c|c|c|c|c|c|c|}
\hline $\begin{array}{l}\text { Similarity } \\
\text { to test set }\end{array}$ & X-Score ${ }^{H M}$ & ChemScore & ASP & L-SVR & $\mathrm{RF}$ & KNN & DT & BRR & MLP \\
\hline 0.92 & $0.619 \pm 0.003$ & $0.582 \pm 0.003$ & $0.617 \pm 0.001$ & $0.696 \pm 0.005$ & $0.799 \pm 0.004$ & $0.670 \pm 0.003$ & $0.649 \pm 0.013$ & $0.710 \pm 0.002$ & $0.551 \pm 0.079$ \\
\hline 0.90 & $0.620 \pm 0.004$ & $0.582 \pm 0.004$ & $0.617 \pm 0.002$ & $0.695 \pm 0.007$ & $0.796 \pm 0.005$ & $0.676 \pm 0.004$ & $0.658 \pm 0.014$ & $0.714 \pm 0.002$ & $0.547 \pm 0.083$ \\
\hline 0.88 & $0.627 \pm 0.003$ & $0.586 \pm 0.004$ & $0.615 \pm 0.003$ & $0.697 \pm 0.006$ & $0.789 \pm 0.006$ & $0.679 \pm 0.005$ & $0.654 \pm 0.013$ & $0.715 \pm 0.003$ & $0.567 \pm 0.089$ \\
\hline 0.86 & $0.624 \pm 0.004$ & $0.588 \pm 0.004$ & $0.609 \pm 0.005$ & $0.679 \pm 0.008$ & $0.779 \pm 0.007$ & $0.679 \pm 0.005$ & $0.641 \pm 0.013$ & $0.709 \pm 0.003$ & $0.549 \pm 0.088$ \\
\hline 0.84 & $0.622 \pm 0.005$ & $0.591 \pm 0.004$ & $0.605 \pm 0.005$ & $0.686 \pm 0.017$ & $0.773 \pm 0.008$ & $0.680 \pm 0.005$ & $0.634 \pm 0.016$ & $0.705 \pm 0.004$ & $0.539 \pm 0.099$ \\
\hline 0.82 & $0.620 \pm 0.005$ & $0.592 \pm 0.005$ & $0.598 \pm 0.006$ & $0.659 \pm 0.033$ & $0.774 \pm 0.009$ & $0.679 \pm 0.005$ & $0.640 \pm 0.015$ & $0.701 \pm 0.004$ & $0.555 \pm 0.081$ \\
\hline 0.80 & $0.615 \pm 0.004$ & $0.594 \pm 0.005$ & $0.595 \pm 0.007$ & $0.668 \pm 0.023$ & $0.771 \pm 0.009$ & $0.678 \pm 0.006$ & $0.640 \pm 0.017$ & $0.697 \pm 0.004$ & $0.558 \pm 0.085$ \\
\hline 0.78 & $0.613 \pm 0.005$ & $0.595 \pm 0.006$ & $0.592 \pm 0.008$ & $0.659 \pm 0.018$ & $0.766 \pm 0.010$ & $0.676 \pm 0.006$ & $0.633 \pm 0.016$ & $0.695 \pm 0.005$ & $0.543 \pm 0.096$ \\
\hline 0.76 & $0.614 \pm 0.005$ & $0.597 \pm 0.006$ & $0.589 \pm 0.009$ & $0.667 \pm 0.017$ & $0.759 \pm 0.013$ & $0.679 \pm 0.006$ & $0.627 \pm 0.019$ & $0.693 \pm 0.005$ & $0.553 \pm 0.098$ \\
\hline 0.74 & $0.615 \pm 0.005$ & $0.602 \pm 0.005$ & $0.589 \pm 0.010$ & $0.668 \pm 0.014$ & $0.755 \pm 0.011$ & $0.675 \pm 0.007$ & $0.622 \pm 0.018$ & $0.693 \pm 0.005$ & $0.540 \pm 0.092$ \\
\hline 0.72 & $0.617 \pm 0.005$ & $0.602 \pm 0.006$ & $0.584 \pm 0.012$ & $0.672 \pm 0.016$ & $0.753 \pm 0.013$ & $0.676 \pm 0.006$ & $0.617 \pm 0.019$ & $0.692 \pm 0.006$ & $0.543 \pm 0.097$ \\
\hline 0.70 & $0.618 \pm 0.005$ & $0.602 \pm 0.006$ & $0.581 \pm 0.011$ & $0.676 \pm 0.015$ & $0.742 \pm 0.016$ & $0.678 \pm 0.006$ & $0.608 \pm 0.021$ & $0.692 \pm 0.005$ & $0.549 \pm 0.087$ \\
\hline 0.68 & $0.619 \pm 0.005$ & $0.608 \pm 0.007$ & $0.587 \pm 0.012$ & $0.666 \pm 0.018$ & $0.737 \pm 0.016$ & $0.677 \pm 0.007$ & $0.598 \pm 0.024$ & $0.686 \pm 0.006$ & $0.520 \pm 0.104$ \\
\hline 0.66 & $0.617 \pm 0.004$ & $0.610 \pm 0.007$ & $0.585 \pm 0.012$ & $0.665 \pm 0.020$ & $0.720 \pm 0.016$ & $0.677 \pm 0.006$ & $0.583 \pm 0.023$ & $0.682 \pm 0.007$ & $0.543 \pm 0.093$ \\
\hline 0.64 & $0.617 \pm 0.005$ & $0.611 \pm 0.006$ & $0.582 \pm 0.011$ & $0.665 \pm 0.017$ & $0.707 \pm 0.015$ & $0.679 \pm 0.006$ & $0.578 \pm 0.022$ & $0.683 \pm 0.006$ & $0.534 \pm 0.088$ \\
\hline 0.62 & $0.620 \pm 0.004$ & $0.619 \pm 0.004$ & $0.592 \pm 0.010$ & $0.661 \pm 0.021$ & $0.711 \pm 0.014$ & $0.683 \pm 0.006$ & $0.581 \pm 0.021$ & $0.682 \pm 0.007$ & $0.505 \pm 0.104$ \\
\hline 0.60 & $0.618 \pm 0.004$ & $0.618 \pm 0.004$ & $0.592 \pm 0.010$ & $0.659 \pm 0.020$ & $0.699 \pm 0.014$ & $0.681 \pm 0.006$ & $0.569 \pm 0.023$ & $0.679 \pm 0.007$ & $0.520 \pm 0.094$ \\
\hline 0.58 & $0.618 \pm 0.004$ & $0.618 \pm 0.004$ & $0.593 \pm 0.010$ & $0.659 \pm 0.022$ & $0.686 \pm 0.015$ & $0.680 \pm 0.006$ & $0.561 \pm 0.025$ & $0.677 \pm 0.007$ & $0.510 \pm 0.108$ \\
\hline 0.56 & $0.618 \pm 0.004$ & $0.619 \pm 0.003$ & $0.594 \pm 0.009$ & $0.660 \pm 0.016$ & $0.681 \pm 0.016$ & $0.683 \pm 0.006$ & $0.562 \pm 0.019$ & $0.676 \pm 0.007$ & $0.514 \pm 0.088$ \\
\hline 0.54 & $0.617 \pm 0.004$ & $0.618 \pm 0.003$ & $0.602 \pm 0.008$ & $0.660 \pm 0.019$ & $0.685 \pm 0.013$ & $0.681 \pm 0.007$ & $0.560 \pm 0.022$ & $0.681 \pm 0.006$ & $0.514 \pm 0.105$ \\
\hline 0.52 & $0.615 \pm 0.004$ & $0.619 \pm 0.003$ & $0.606 \pm 0.008$ & $0.649 \pm 0.024$ & $0.669 \pm 0.013$ & $0.676 \pm 0.006$ & $0.542 \pm 0.025$ & $0.672 \pm 0.008$ & $0.528 \pm 0.089$ \\
\hline
\end{tabular}


PART 2. Performance of all models calibrated on the training sets with different levels of similarity by ligand shape

Table S4. Pearson correlation coefficients produced by all models on the CASF-2016 test set

\begin{tabular}{|c|c|c|c|c|c|c|c|c|c|}
\hline $\begin{array}{l}\text { Similarity } \\
\text { to test set }\end{array}$ & X-Score ${ }^{H M}$ & ChemScore & ASP & L-SVR & $\mathrm{RF}$ & $\mathrm{KNN}$ & DT & BRR & MLP \\
\hline 0.67 & $0.645 \pm 0.001$ & $0.616 \pm 0.002$ & $0.488 \pm 0.000$ & $0.637 \pm 0.011$ & $0.754 \pm 0.007$ & $0.682 \pm 0.003$ & $0.597 \pm 0.014$ & $0.639 \pm 0.006$ & $0.516 \pm 0.097$ \\
\hline 0.66 & $0.643 \pm 0.001$ & $0.613 \pm 0.003$ & $0.488 \pm 0.001$ & $0.629 \pm 0.017$ & $0.760 \pm 0.009$ & $0.680 \pm 0.004$ & $0.604 \pm 0.018$ & $0.651 \pm 0.006$ & $0.527 \pm 0.084$ \\
\hline 0.65 & $0.641 \pm 0.002$ & $0.609 \pm 0.004$ & $0.487 \pm 0.001$ & $0.632 \pm 0.023$ & $0.759 \pm 0.010$ & $0.678 \pm 0.005$ & $0.609 \pm 0.020$ & $0.659 \pm 0.008$ & $0.513 \pm 0.087$ \\
\hline 0.64 & $0.640 \pm 0.003$ & $0.608 \pm 0.005$ & $0.486 \pm 0.002$ & $0.634 \pm 0.022$ & $0.755 \pm 0.012$ & $0.677 \pm 0.005$ & $0.611 \pm 0.019$ & $0.661 \pm 0.008$ & $0.528 \pm 0.087$ \\
\hline 0.63 & $0.636 \pm 0.004$ & $0.604 \pm 0.007$ & $0.484 \pm 0.003$ & $0.634 \pm 0.021$ & $0.748 \pm 0.014$ & $0.676 \pm 0.005$ & $0.606 \pm 0.019$ & $0.659 \pm 0.008$ & $0.534 \pm 0.092$ \\
\hline 0.62 & $0.631 \pm 0.005$ & $0.606 \pm 0.005$ & $0.481 \pm 0.004$ & $0.636 \pm 0.021$ & $0.746 \pm 0.012$ & $0.674 \pm 0.005$ & $0.603 \pm 0.021$ & $0.660 \pm 0.009$ & $0.524 \pm 0.093$ \\
\hline 0.61 & $0.628 \pm 0.005$ & $0.607 \pm 0.006$ & $0.479 \pm 0.004$ & $0.633 \pm 0.021$ & $0.746 \pm 0.013$ & $0.675 \pm 0.006$ & $0.602 \pm 0.018$ & $0.659 \pm 0.009$ & $0.530 \pm 0.094$ \\
\hline 0.60 & $0.624 \pm 0.006$ & $0.603 \pm 0.005$ & $0.478 \pm 0.004$ & $0.634 \pm 0.021$ & $0.741 \pm 0.015$ & $0.678 \pm 0.007$ & $0.597 \pm 0.021$ & $0.658 \pm 0.008$ & $0.537 \pm 0.089$ \\
\hline 0.59 & $0.621 \pm 0.007$ & $0.602 \pm 0.006$ & $0.476 \pm 0.005$ & $0.632 \pm 0.018$ & $0.727 \pm 0.015$ & $0.675 \pm 0.006$ & $0.587 \pm 0.020$ & $0.653 \pm 0.008$ & $0.546 \pm 0.090$ \\
\hline 0.58 & $0.619 \pm 0.007$ & $0.600 \pm 0.006$ & $0.472 \pm 0.006$ & $0.636 \pm 0.018$ & $0.732 \pm 0.012$ & $0.675 \pm 0.007$ & $0.591 \pm 0.018$ & $0.657 \pm 0.007$ & $0.530 \pm 0.094$ \\
\hline 0.57 & $0.616 \pm 0.005$ & $0.598 \pm 0.005$ & $0.469 \pm 0.006$ & $0.633 \pm 0.015$ & $0.722 \pm 0.012$ & $0.671 \pm 0.005$ & $0.578 \pm 0.019$ & $0.664 \pm 0.006$ & $0.545 \pm 0.100$ \\
\hline 0.56 & $0.614 \pm 0.004$ & $0.599 \pm 0.004$ & $0.467 \pm 0.006$ & $0.646 \pm 0.014$ & $0.705 \pm 0.012$ & $0.668 \pm 0.005$ & $0.561 \pm 0.016$ & $0.667 \pm 0.004$ & $0.532 \pm 0.094$ \\
\hline 0.55 & $0.607 \pm 0.004$ & $0.600 \pm 0.003$ & $0.460 \pm 0.003$ & $0.647 \pm 0.007$ & $0.689 \pm 0.008$ & $0.668 \pm 0.003$ & $0.553 \pm 0.015$ & $0.668 \pm 0.003$ & $0.536 \pm 0.091$ \\
\hline
\end{tabular}


Table S5. RMSE produced by all models on the CASF-2016 test set

\begin{tabular}{|c|c|c|c|c|c|c|c|c|c|}
\hline $\begin{array}{l}\text { Similarity } \\
\text { to test set }\end{array}$ & X-Score ${ }^{\mathrm{HM}}$ & ChemScore & ASP & L-SVR & RF & KNN & DT & BRR & MLP \\
\hline 0.67 & $1.71 \pm 0.00$ & $1.73 \pm 0.00$ & $1.92 \pm 0.00$ & $2.26 \pm 0.11$ & $1.49 \pm 0.01$ & $1.64 \pm 0.01$ & $1.75 \pm 0.03$ & $1.73 \pm 0.01$ & $3.84 \pm 1.02$ \\
\hline 0.66 & $1.72 \pm 0.00$ & $1.74 \pm 0.01$ & $1.92 \pm 0.01$ & $2.28 \pm 0.13$ & $1.48 \pm 0.02$ & $1.64 \pm 0.01$ & $1.74 \pm 0.03$ & $1.71 \pm 0.01$ & $3.73 \pm 0.85$ \\
\hline 0.65 & $1.72 \pm 0.01$ & $1.75 \pm 0.01$ & $1.91 \pm 0.01$ & $2.26 \pm 0.12$ & $1.48 \pm 0.02$ & $1.64 \pm 0.01$ & $1.73 \pm 0.03$ & $1.69 \pm 0.01$ & $3.94 \pm 0.88$ \\
\hline 0.64 & $1.73 \pm 0.01$ & $1.76 \pm 0.01$ & $1.92 \pm 0.01$ & $2.24 \pm 0.13$ & $1.49 \pm 0.02$ & $1.65 \pm 0.01$ & $1.73 \pm 0.03$ & $1.69 \pm 0.01$ & $3.81 \pm 0.90$ \\
\hline 0.63 & $1.74 \pm 0.01$ & $1.76 \pm 0.01$ & $1.93 \pm 0.01$ & $2.26 \pm 0.12$ & $1.50 \pm 0.03$ & $1.66 \pm 0.01$ & $1.74 \pm 0.03$ & $1.70 \pm 0.01$ & $3.76 \pm 0.94$ \\
\hline 0.62 & $1.74 \pm 0.01$ & $1.77 \pm 0.01$ & $1.93 \pm 0.01$ & $2.28 \pm 0.12$ & $1.51 \pm 0.02$ & $1.66 \pm 0.01$ & $1.74 \pm 0.04$ & $1.70 \pm 0.01$ & $3.92 \pm 1.06$ \\
\hline 0.61 & $1.75 \pm 0.01$ & $1.77 \pm 0.01$ & $1.94 \pm 0.01$ & $2.26 \pm 0.14$ & $1.51 \pm 0.03$ & $1.67 \pm 0.01$ & $1.74 \pm 0.03$ & $1.70 \pm 0.02$ & $3.95 \pm 0.96$ \\
\hline 0.60 & $1.76 \pm 0.01$ & $1.78 \pm 0.01$ & $1.95 \pm 0.01$ & $2.27 \pm 0.12$ & $1.52 \pm 0.03$ & $1.67 \pm 0.01$ & $1.75 \pm 0.04$ & $1.71 \pm 0.01$ & $3.69 \pm 0.87$ \\
\hline 0.59 & $1.77 \pm 0.01$ & $1.80 \pm 0.01$ & $1.96 \pm 0.01$ & $2.28 \pm 0.10$ & $1.54 \pm 0.03$ & $1.68 \pm 0.01$ & $1.76 \pm 0.03$ & $1.72 \pm 0.01$ & $3.74 \pm 0.85$ \\
\hline 0.58 & $1.78 \pm 0.01$ & $1.81 \pm 0.01$ & $1.98 \pm 0.01$ & $2.29 \pm 0.12$ & $1.53 \pm 0.02$ & $1.69 \pm 0.01$ & $1.76 \pm 0.03$ & $1.72 \pm 0.01$ & $3.84 \pm 0.89$ \\
\hline 0.57 & $1.78 \pm 0.01$ & $1.81 \pm 0.01$ & $1.99 \pm 0.01$ & $2.29 \pm 0.10$ & $1.55 \pm 0.02$ & $1.71 \pm 0.01$ & $1.78 \pm 0.03$ & $1.72 \pm 0.01$ & $3.86 \pm 1.08$ \\
\hline 0.56 & $1.78 \pm 0.01$ & $1.82 \pm 0.01$ & $2.00 \pm 0.01$ & $2.24 \pm 0.11$ & $1.58 \pm 0.02$ & $1.72 \pm 0.01$ & $1.81 \pm 0.03$ & $1.72 \pm 0.01$ & $3.93 \pm 0.92$ \\
\hline 0.55 & $1.78 \pm 0.01$ & $1.81 \pm 0.01$ & $1.98 \pm 0.00$ & $2.22 \pm 0.08$ & $1.61 \pm 0.01$ & $1.73 \pm 0.01$ & $1.82 \pm 0.03$ & $1.72 \pm 0.01$ & $3.89 \pm 0.79$ \\
\hline
\end{tabular}

Table S6. Spearman correlation coefficients produced by all models on the CASF-2016 test set

\begin{tabular}{|c|c|c|c|c|c|c|c|c|c|}
\hline $\begin{array}{l}\text { Similarity } \\
\text { to test set }\end{array}$ & X-Score $\mathrm{HM}$ & ChemScore & ASP & L-SVR & $\mathrm{RF}$ & KNN & DT & BRR & MLP \\
\hline 0.67 & $0.637 \pm 0.002$ & $0.615 \pm 0.003$ & $0.618 \pm 0.002$ & $0.670 \pm 0.011$ & $0.738 \pm 0.010$ & $0.694 \pm 0.003$ & $0.594 \pm 0.016$ & $0.673 \pm 0.005$ & $0.527 \pm 0.099$ \\
\hline 0.66 & $0.635 \pm 0.002$ & $0.615 \pm 0.003$ & $0.616 \pm 0.004$ & $0.660 \pm 0.016$ & $0.749 \pm 0.010$ & $0.687 \pm 0.004$ & $0.601 \pm 0.020$ & $0.683 \pm 0.005$ & $0.538 \pm 0.085$ \\
\hline 0.65 & $0.632 \pm 0.003$ & $0.610 \pm 0.005$ & $0.613 \pm 0.004$ & $0.662 \pm 0.021$ & $0.750 \pm 0.011$ & $0.684 \pm 0.006$ & $0.608 \pm 0.020$ & $0.690 \pm 0.006$ & $0.525 \pm 0.089$ \\
\hline 0.64 & $0.631 \pm 0.003$ & $0.609 \pm 0.005$ & $0.610 \pm 0.006$ & $0.662 \pm 0.020$ & $0.749 \pm 0.013$ & $0.682 \pm 0.005$ & $0.613 \pm 0.020$ & $0.690 \pm 0.006$ & $0.541 \pm 0.089$ \\
\hline 0.63 & $0.625 \pm 0.005$ & $0.606 \pm 0.007$ & $0.603 \pm 0.008$ & $0.663 \pm 0.019$ & $0.741 \pm 0.015$ & $0.679 \pm 0.006$ & $0.606 \pm 0.020$ & $0.687 \pm 0.006$ & $0.546 \pm 0.094$ \\
\hline 0.62 & $0.620 \pm 0.005$ & $0.608 \pm 0.006$ & $0.594 \pm 0.010$ & $0.666 \pm 0.017$ & $0.739 \pm 0.012$ & $0.677 \pm 0.005$ & $0.605 \pm 0.021$ & $0.686 \pm 0.007$ & $0.538 \pm 0.096$ \\
\hline 0.61 & $0.617 \pm 0.005$ & $0.609 \pm 0.005$ & $0.588 \pm 0.010$ & $0.663 \pm 0.019$ & $0.740 \pm 0.015$ & $0.677 \pm 0.005$ & $0.605 \pm 0.019$ & $0.685 \pm 0.007$ & $0.543 \pm 0.094$ \\
\hline 0.60 & $0.613 \pm 0.005$ & $0.606 \pm 0.005$ & $0.588 \pm 0.010$ & $0.663 \pm 0.018$ & $0.735 \pm 0.016$ & $0.679 \pm 0.007$ & $0.601 \pm 0.021$ & $0.682 \pm 0.007$ & $0.551 \pm 0.091$ \\
\hline 0.59 & $0.610 \pm 0.006$ & $0.608 \pm 0.006$ & $0.585 \pm 0.013$ & $0.662 \pm 0.016$ & $0.723 \pm 0.016$ & $0.676 \pm 0.007$ & $0.590 \pm 0.022$ & $0.677 \pm 0.006$ & $0.559 \pm 0.092$ \\
\hline 0.58 & $0.609 \pm 0.007$ & $0.609 \pm 0.005$ & $0.576 \pm 0.015$ & $0.662 \pm 0.017$ & $0.730 \pm 0.013$ & $0.676 \pm 0.007$ & $0.593 \pm 0.018$ & $0.679 \pm 0.006$ & $0.545 \pm 0.095$ \\
\hline 0.57 & $0.606 \pm 0.005$ & $0.607 \pm 0.005$ & $0.570 \pm 0.013$ & $0.662 \pm 0.014$ & $0.722 \pm 0.012$ & $0.673 \pm 0.005$ & $0.581 \pm 0.020$ & $0.682 \pm 0.005$ & $0.561 \pm 0.103$ \\
\hline 0.56 & $0.605 \pm 0.004$ & $0.606 \pm 0.004$ & $0.565 \pm 0.013$ & $0.677 \pm 0.011$ & $0.704 \pm 0.013$ & $0.671 \pm 0.006$ & $0.567 \pm 0.016$ & $0.685 \pm 0.003$ & $0.548 \pm 0.096$ \\
\hline 0.55 & $0.600 \pm 0.004$ & $0.605 \pm 0.003$ & $0.551 \pm 0.007$ & $0.681 \pm 0.005$ & $0.684 \pm 0.009$ & $0.671 \pm 0.004$ & $0.557 \pm 0.016$ & $0.684 \pm 0.003$ & $0.552 \pm 0.093$ \\
\hline
\end{tabular}


PART 3. Performance of all models calibrated on the training sets with different levels of similarity by binding pocket

Table S7. Pearson correlation coefficients produced by all models on the CASF-2016 test set

\begin{tabular}{|c|c|c|c|c|c|c|c|c|c|}
\hline $\begin{array}{l}\text { Similarity } \\
\text { to test set }\end{array}$ & X-Score ${ }^{H M}$ & ChemScore & ASP & L-SVR & RF & KNN & DT & BRR & MLP \\
\hline 0.84 & $0.609 \pm 0.006$ & $0.584 \pm 0.005$ & $0.468 \pm 0.003$ & $0.615 \pm 0.023$ & $0.769 \pm 0.007$ & $0.675 \pm 0.003$ & $0.611 \pm 0.015$ & $0.661 \pm 0.004$ & $0.547 \pm 0.088$ \\
\hline 0.83 & $0.618 \pm 0.006$ & $0.588 \pm 0.005$ & $0.471 \pm 0.004$ & $0.603 \pm 0.020$ & $0.767 \pm 0.008$ & $0.684 \pm 0.006$ & $0.608 \pm 0.019$ & $0.663 \pm 0.005$ & $0.538 \pm 0.093$ \\
\hline 0.82 & $0.624 \pm 0.005$ & $0.593 \pm 0.006$ & $0.474 \pm 0.004$ & $0.603 \pm 0.025$ & $0.764 \pm 0.011$ & $0.684 \pm 0.005$ & $0.610 \pm 0.020$ & $0.666 \pm 0.005$ & $0.544 \pm 0.092$ \\
\hline 0.81 & $0.625 \pm 0.005$ & $0.598 \pm 0.006$ & $0.477 \pm 0.005$ & $0.616 \pm 0.024$ & $0.767 \pm 0.009$ & $0.684 \pm 0.005$ & $0.616 \pm 0.018$ & $0.668 \pm 0.006$ & $0.543 \pm 0.095$ \\
\hline 0.80 & $0.628 \pm 0.005$ & $0.602 \pm 0.007$ & $0.478 \pm 0.005$ & $0.624 \pm 0.024$ & $0.761 \pm 0.011$ & $0.681 \pm 0.005$ & $0.616 \pm 0.018$ & $0.668 \pm 0.007$ & $0.519 \pm 0.092$ \\
\hline 0.79 & $0.629 \pm 0.005$ & $0.606 \pm 0.006$ & $0.478 \pm 0.004$ & $0.626 \pm 0.022$ & $0.755 \pm 0.012$ & $0.679 \pm 0.005$ & $0.613 \pm 0.019$ & $0.665 \pm 0.007$ & $0.524 \pm 0.081$ \\
\hline 0.78 & $0.630 \pm 0.005$ & $0.607 \pm 0.006$ & $0.477 \pm 0.004$ & $0.627 \pm 0.023$ & $0.749 \pm 0.013$ & $0.677 \pm 0.006$ & $0.606 \pm 0.022$ & $0.663 \pm 0.008$ & $0.526 \pm 0.090$ \\
\hline 0.77 & $0.629 \pm 0.005$ & $0.609 \pm 0.005$ & $0.478 \pm 0.004$ & $0.635 \pm 0.023$ & $0.737 \pm 0.014$ & $0.675 \pm 0.007$ & $0.593 \pm 0.022$ & $0.662 \pm 0.008$ & $0.529 \pm 0.086$ \\
\hline 0.76 & $0.630 \pm 0.005$ & $0.613 \pm 0.006$ & $0.479 \pm 0.004$ & $0.630 \pm 0.023$ & $0.731 \pm 0.014$ & $0.674 \pm 0.006$ & $0.591 \pm 0.017$ & $0.664 \pm 0.008$ & $0.531 \pm 0.083$ \\
\hline 0.75 & $0.632 \pm 0.004$ & $0.619 \pm 0.005$ & $0.481 \pm 0.003$ & $0.623 \pm 0.022$ & $0.715 \pm 0.013$ & $0.673 \pm 0.005$ & $0.579 \pm 0.019$ & $0.659 \pm 0.009$ & $0.524 \pm 0.092$ \\
\hline 0.74 & $0.631 \pm 0.005$ & $0.620 \pm 0.004$ & $0.483 \pm 0.003$ & $0.629 \pm 0.019$ & $0.711 \pm 0.013$ & $0.670 \pm 0.006$ & $0.576 \pm 0.020$ & $0.658 \pm 0.009$ & $0.523 \pm 0.090$ \\
\hline 0.73 & $0.632 \pm 0.004$ & $0.622 \pm 0.003$ & $0.484 \pm 0.002$ & $0.625 \pm 0.020$ & $0.703 \pm 0.011$ & $0.671 \pm 0.005$ & $0.572 \pm 0.016$ & $0.660 \pm 0.007$ & $0.505 \pm 0.097$ \\
\hline 0.72 & $0.632 \pm 0.003$ & $0.622 \pm 0.003$ & $0.485 \pm 0.001$ & $0.621 \pm 0.021$ & $0.694 \pm 0.011$ & $0.667 \pm 0.005$ & $0.563 \pm 0.017$ & $0.658 \pm 0.007$ & $0.507 \pm 0.095$ \\
\hline 0.71 & $0.632 \pm 0.003$ & $0.625 \pm 0.003$ & $0.486 \pm 0.001$ & $0.630 \pm 0.017$ & $0.695 \pm 0.010$ & $0.659 \pm 0.004$ & $0.557 \pm 0.018$ & $0.658 \pm 0.005$ & $0.514 \pm 0.106$ \\
\hline 0.70 & $0.630 \pm 0.002$ & $0.627 \pm 0.001$ & $0.486 \pm 0.001$ & $0.653 \pm 0.007$ & $0.701 \pm 0.005$ & $0.661 \pm 0.003$ & $0.567 \pm 0.013$ & $0.658 \pm 0.004$ & $0.497 \pm 0.095$ \\
\hline
\end{tabular}


Table S8. RMSE produced by all models on the CASF-2016 test set

\begin{tabular}{|c|c|c|c|c|c|c|c|c|c|}
\hline $\begin{array}{l}\text { Similarity } \\
\text { to test set }\end{array}$ & X-Score ${ }^{H M}$ & ChemScore & ASP & L-SVR & $\mathrm{RF}$ & KNN & DT & BRR & MLP \\
\hline 0.84 & $1.82 \pm 0.01$ & $1.83 \pm 0.01$ & $1.97 \pm 0.01$ & $2.37 \pm 0.09$ & $1.46 \pm 0.02$ & $1.72 \pm 0.01$ & $1.72 \pm 0.03$ & $1.70 \pm 0.01$ & $3.72 \pm 0.89$ \\
\hline 0.83 & $1.79 \pm 0.01$ & $1.81 \pm 0.01$ & $1.95 \pm 0.01$ & $2.35 \pm 0.10$ & $1.47 \pm 0.02$ & $1.70 \pm 0.01$ & $1.73 \pm 0.03$ & $1.69 \pm 0.01$ & $3.84 \pm 0.98$ \\
\hline 0.82 & $1.78 \pm 0.01$ & $1.80 \pm 0.01$ & $1.94 \pm 0.01$ & $2.34 \pm 0.13$ & $1.48 \pm 0.02$ & $1.68 \pm 0.01$ & $1.72 \pm 0.04$ & $1.69 \pm 0.01$ & $3.75 \pm 0.91$ \\
\hline 0.81 & $1.76 \pm 0.01$ & $1.78 \pm 0.01$ & $1.94 \pm 0.01$ & $2.29 \pm 0.13$ & $1.47 \pm 0.02$ & $1.67 \pm 0.01$ & $1.71 \pm 0.03$ & $1.69 \pm 0.01$ & $3.81 \pm 1.00$ \\
\hline 0.80 & $1.75 \pm 0.01$ & $1.77 \pm 0.01$ & $1.94 \pm 0.01$ & $2.26 \pm 0.11$ & $1.48 \pm 0.02$ & $1.66 \pm 0.01$ & $1.72 \pm 0.03$ & $1.68 \pm 0.01$ & $4.06 \pm 0.98$ \\
\hline 0.79 & $1.74 \pm 0.01$ & $1.76 \pm 0.01$ & $1.93 \pm 0.01$ & $2.26 \pm 0.12$ & $1.48 \pm 0.02$ & $1.66 \pm 0.01$ & $1.72 \pm 0.04$ & $1.68 \pm 0.01$ & $3.96 \pm 0.95$ \\
\hline 0.78 & $1.74 \pm 0.01$ & $1.76 \pm 0.01$ & $1.93 \pm 0.01$ & $2.24 \pm 0.12$ & $1.50 \pm 0.03$ & $1.66 \pm 0.01$ & $1.73 \pm 0.04$ & $1.69 \pm 0.01$ & $3.94 \pm 0.99$ \\
\hline 0.77 & $1.74 \pm 0.01$ & $1.76 \pm 0.01$ & $1.94 \pm 0.01$ & $2.23 \pm 0.13$ & $1.52 \pm 0.03$ & $1.67 \pm 0.01$ & $1.76 \pm 0.04$ & $1.70 \pm 0.01$ & $3.97 \pm 1.00$ \\
\hline 0.76 & $1.74 \pm 0.01$ & $1.75 \pm 0.01$ & $1.95 \pm 0.01$ & $2.26 \pm 0.12$ & $1.53 \pm 0.03$ & $1.66 \pm 0.01$ & $1.76 \pm 0.03$ & $1.70 \pm 0.01$ & $3.96 \pm 1.06$ \\
\hline 0.75 & $1.73 \pm 0.01$ & $1.74 \pm 0.01$ & $1.96 \pm 0.02$ & $2.28 \pm 0.13$ & $1.56 \pm 0.02$ & $1.66 \pm 0.01$ & $1.78 \pm 0.03$ & $1.71 \pm 0.01$ & $4.01 \pm 1.10$ \\
\hline 0.74 & $1.73 \pm 0.01$ & $1.74 \pm 0.01$ & $1.97 \pm 0.01$ & $2.29 \pm 0.12$ & $1.57 \pm 0.02$ & $1.67 \pm 0.01$ & $1.79 \pm 0.03$ & $1.72 \pm 0.01$ & $3.94 \pm 0.90$ \\
\hline 0.73 & $1.74 \pm 0.01$ & $1.75 \pm 0.01$ & $1.99 \pm 0.02$ & $2.31 \pm 0.14$ & $1.59 \pm 0.02$ & $1.67 \pm 0.01$ & $1.80 \pm 0.03$ & $1.73 \pm 0.01$ & $4.00 \pm 0.97$ \\
\hline 0.72 & $1.74 \pm 0.01$ & $1.75 \pm 0.01$ & $2.00 \pm 0.01$ & $2.32 \pm 0.14$ & $1.60 \pm 0.02$ & $1.67 \pm 0.01$ & $1.81 \pm 0.03$ & $1.73 \pm 0.01$ & $4.00 \pm 0.92$ \\
\hline 0.71 & $1.74 \pm 0.01$ & $1.75 \pm 0.01$ & $2.01 \pm 0.01$ & $2.28 \pm 0.13$ & $1.60 \pm 0.02$ & $1.69 \pm 0.01$ & $1.82 \pm 0.03$ & $1.74 \pm 0.01$ & $3.92 \pm 0.92$ \\
\hline 0.70 & $1.74 \pm 0.01$ & $1.74 \pm 0.00$ & $2.02 \pm 0.01$ & $2.16 \pm 0.10$ & $1.59 \pm 0.01$ & $1.69 \pm 0.01$ & $1.81 \pm 0.02$ & $1.74 \pm 0.01$ & $4.06 \pm 0.88$ \\
\hline
\end{tabular}


Table S9. Spearman correlation coefficients produced by all models on the CASF-2016 test set

\begin{tabular}{|c|c|c|c|c|c|c|c|c|c|}
\hline $\begin{array}{l}\text { Similarity } \\
\text { to test set }\end{array}$ & $\mathrm{X}$-Score ${ }^{\mathrm{HM}}$ & ChemScore & ASP & L-SVR & RF & KNN & DT & BRR & MLP \\
\hline 0.84 & $0.597 \pm 0.006$ & $0.584 \pm 0.005$ & $0.564 \pm 0.006$ & $0.644 \pm 0.021$ & $0.757 \pm 0.009$ & $0.668 \pm 0.004$ & $0.596 \pm 0.020$ & $0.686 \pm 0.003$ & $0.562 \pm 0.090$ \\
\hline 0.83 & $0.606 \pm 0.006$ & $0.591 \pm 0.006$ & $0.569 \pm 0.009$ & $0.635 \pm 0.021$ & $0.760 \pm 0.010$ & $0.679 \pm 0.007$ & $0.603 \pm 0.018$ & $0.688 \pm 0.004$ & $0.553 \pm 0.095$ \\
\hline 0.82 & $0.613 \pm 0.005$ & $0.597 \pm 0.006$ & $0.579 \pm 0.010$ & $0.639 \pm 0.024$ & $0.760 \pm 0.013$ & $0.682 \pm 0.006$ & $0.607 \pm 0.022$ & $0.691 \pm 0.004$ & $0.558 \pm 0.095$ \\
\hline 0.81 & $0.614 \pm 0.005$ & $0.603 \pm 0.005$ & $0.584 \pm 0.012$ & $0.653 \pm 0.022$ & $0.764 \pm 0.010$ & $0.683 \pm 0.006$ & $0.616 \pm 0.018$ & $0.693 \pm 0.005$ & $0.557 \pm 0.097$ \\
\hline 0.80 & $0.617 \pm 0.005$ & $0.606 \pm 0.007$ & $0.586 \pm 0.012$ & $0.659 \pm 0.019$ & $0.758 \pm 0.012$ & $0.681 \pm 0.005$ & $0.616 \pm 0.019$ & $0.693 \pm 0.006$ & $0.533 \pm 0.094$ \\
\hline 0.79 & $0.618 \pm 0.005$ & $0.607 \pm 0.006$ & $0.585 \pm 0.010$ & $0.660 \pm 0.020$ & $0.751 \pm 0.014$ & $0.680 \pm 0.006$ & $0.616 \pm 0.020$ & $0.691 \pm 0.005$ & $0.538 \pm 0.082$ \\
\hline 0.78 & $0.619 \pm 0.005$ & $0.608 \pm 0.006$ & $0.584 \pm 0.011$ & $0.659 \pm 0.021$ & $0.743 \pm 0.014$ & $0.678 \pm 0.006$ & $0.609 \pm 0.023$ & $0.689 \pm 0.006$ & $0.540 \pm 0.092$ \\
\hline 0.77 & $0.618 \pm 0.005$ & $0.609 \pm 0.006$ & $0.587 \pm 0.011$ & $0.665 \pm 0.021$ & $0.732 \pm 0.016$ & $0.678 \pm 0.006$ & $0.598 \pm 0.021$ & $0.687 \pm 0.007$ & $0.543 \pm 0.088$ \\
\hline 0.76 & $0.620 \pm 0.005$ & $0.612 \pm 0.005$ & $0.590 \pm 0.010$ & $0.660 \pm 0.022$ & $0.728 \pm 0.015$ & $0.678 \pm 0.006$ & $0.596 \pm 0.017$ & $0.687 \pm 0.007$ & $0.544 \pm 0.084$ \\
\hline 0.75 & $0.621 \pm 0.003$ & $0.617 \pm 0.005$ & $0.596 \pm 0.009$ & $0.655 \pm 0.020$ & $0.717 \pm 0.013$ & $0.678 \pm 0.005$ & $0.587 \pm 0.020$ & $0.683 \pm 0.007$ & $0.537 \pm 0.094$ \\
\hline 0.74 & $0.621 \pm 0.004$ & $0.618 \pm 0.004$ & $0.601 \pm 0.008$ & $0.660 \pm 0.017$ & $0.715 \pm 0.013$ & $0.675 \pm 0.006$ & $0.583 \pm 0.020$ & $0.682 \pm 0.007$ & $0.535 \pm 0.092$ \\
\hline 0.73 & $0.621 \pm 0.003$ & $0.618 \pm 0.003$ & $0.604 \pm 0.005$ & $0.655 \pm 0.020$ & $0.707 \pm 0.013$ & $0.676 \pm 0.005$ & $0.580 \pm 0.016$ & $0.684 \pm 0.005$ & $0.517 \pm 0.099$ \\
\hline 0.72 & $0.622 \pm 0.003$ & $0.618 \pm 0.003$ & $0.608 \pm 0.005$ & $0.652 \pm 0.021$ & $0.705 \pm 0.011$ & $0.672 \pm 0.005$ & $0.573 \pm 0.017$ & $0.682 \pm 0.005$ & $0.519 \pm 0.097$ \\
\hline 0.71 & $0.622 \pm 0.003$ & $0.619 \pm 0.002$ & $0.608 \pm 0.004$ & $0.661 \pm 0.016$ & $0.709 \pm 0.011$ & $0.666 \pm 0.004$ & $0.569 \pm 0.018$ & $0.683 \pm 0.004$ & $0.523 \pm 0.108$ \\
\hline 0.70 & $0.620 \pm 0.002$ & $0.621 \pm 0.001$ & $0.611 \pm 0.002$ & $0.681 \pm 0.007$ & $0.714 \pm 0.005$ & $0.669 \pm 0.004$ & $0.578 \pm 0.014$ & $0.683 \pm 0.003$ & $0.508 \pm 0.097$ \\
\hline
\end{tabular}


PART 4. Performance of all models calibrated on the training sets with different levels of similarity by the 3-in-1 combined metric

Table S10. Pearson correlation coefficients produced by all models on the CASF-2016 test set

\begin{tabular}{|c|c|c|c|c|c|c|c|c|c|}
\hline $\begin{array}{l}\text { Similarity } \\
\text { to test set }\end{array}$ & X-Score ${ }^{\mathrm{HM}}$ & ChemScore & ASP & L-SVR & RF & KNN & DT & BRR & MLP \\
\hline 0.73 & $0.612 \pm 0.002$ & $0.579 \pm 0.001$ & $0.488 \pm 0.000$ & $0.652 \pm 0.008$ & $0.805 \pm 0.002$ & $0.679 \pm 0.001$ & $0.654 \pm 0.008$ & $0.680 \pm 0.001$ & $0.553 \pm 0.074$ \\
\hline 0.72 & $0.611 \pm 0.004$ & $0.580 \pm 0.003$ & $0.486 \pm 0.001$ & $0.640 \pm 0.017$ & $0.800 \pm 0.005$ & $0.677 \pm 0.003$ & $0.650 \pm 0.011$ & $0.676 \pm 0.002$ & $0.547 \pm 0.074$ \\
\hline 0.71 & $0.609 \pm 0.005$ & $0.579 \pm 0.005$ & $0.481 \pm 0.003$ & $0.641 \pm 0.022$ & $0.792 \pm 0.008$ & $0.677 \pm 0.003$ & $0.646 \pm 0.015$ & $0.674 \pm 0.004$ & $0.542 \pm 0.095$ \\
\hline 0.70 & $0.613 \pm 0.006$ & $0.581 \pm 0.005$ & $0.472 \pm 0.004$ & $0.618 \pm 0.023$ & $0.778 \pm 0.009$ & $0.673 \pm 0.005$ & $0.642 \pm 0.014$ & $0.668 \pm 0.004$ & $0.540 \pm 0.096$ \\
\hline 0.69 & $0.621 \pm 0.007$ & $0.589 \pm 0.007$ & $0.472 \pm 0.005$ & $0.626 \pm 0.022$ & $0.771 \pm 0.009$ & $0.674 \pm 0.005$ & $0.641 \pm 0.015$ & $0.669 \pm 0.005$ & $0.540 \pm 0.089$ \\
\hline 0.68 & $0.628 \pm 0.005$ & $0.593 \pm 0.007$ & $0.471 \pm 0.005$ & $0.645 \pm 0.024$ & $0.765 \pm 0.011$ & $0.674 \pm 0.005$ & $0.632 \pm 0.018$ & $0.669 \pm 0.006$ & $0.536 \pm 0.087$ \\
\hline 0.67 & $0.628 \pm 0.005$ & $0.596 \pm 0.007$ & $0.468 \pm 0.006$ & $0.648 \pm 0.023$ & $0.761 \pm 0.011$ & $0.672 \pm 0.005$ & $0.627 \pm 0.019$ & $0.666 \pm 0.006$ & $0.535 \pm 0.094$ \\
\hline 0.66 & $0.628 \pm 0.005$ & $0.603 \pm 0.006$ & $0.471 \pm 0.006$ & $0.639 \pm 0.024$ & $0.754 \pm 0.011$ & $0.673 \pm 0.006$ & $0.618 \pm 0.017$ & $0.665 \pm 0.008$ & $0.530 \pm 0.088$ \\
\hline 0.65 & $0.629 \pm 0.005$ & $0.608 \pm 0.006$ & $0.475 \pm 0.006$ & $0.631 \pm 0.022$ & $0.750 \pm 0.013$ & $0.674 \pm 0.006$ & $0.606 \pm 0.019$ & $0.663 \pm 0.008$ & $0.529 \pm 0.095$ \\
\hline 0.64 & $0.629 \pm 0.005$ & $0.608 \pm 0.006$ & $0.478 \pm 0.005$ & $0.634 \pm 0.022$ & $0.745 \pm 0.013$ & $0.674 \pm 0.006$ & $0.602 \pm 0.019$ & $0.661 \pm 0.008$ & $0.528 \pm 0.089$ \\
\hline 0.63 & $0.629 \pm 0.005$ & $0.607 \pm 0.006$ & $0.477 \pm 0.005$ & $0.633 \pm 0.020$ & $0.732 \pm 0.013$ & $0.674 \pm 0.006$ & $0.593 \pm 0.019$ & $0.663 \pm 0.008$ & $0.531 \pm 0.091$ \\
\hline 0.62 & $0.627 \pm 0.006$ & $0.607 \pm 0.007$ & $0.476 \pm 0.005$ & $0.632 \pm 0.020$ & $0.714 \pm 0.015$ & $0.672 \pm 0.006$ & $0.581 \pm 0.019$ & $0.667 \pm 0.008$ & $0.516 \pm 0.090$ \\
\hline 0.61 & $0.626 \pm 0.006$ & $0.613 \pm 0.005$ & $0.478 \pm 0.004$ & $0.630 \pm 0.025$ & $0.710 \pm 0.013$ & $0.671 \pm 0.007$ & $0.572 \pm 0.020$ & $0.673 \pm 0.007$ & $0.523 \pm 0.094$ \\
\hline 0.60 & $0.627 \pm 0.005$ & $0.617 \pm 0.005$ & $0.478 \pm 0.003$ & $0.628 \pm 0.024$ & $0.695 \pm 0.014$ & $0.668 \pm 0.007$ & $0.568 \pm 0.019$ & $0.674 \pm 0.006$ & $0.512 \pm 0.101$ \\
\hline 0.59 & $0.626 \pm 0.005$ & $0.618 \pm 0.005$ & $0.480 \pm 0.003$ & $0.626 \pm 0.020$ & $0.684 \pm 0.013$ & $0.659 \pm 0.006$ & $0.546 \pm 0.022$ & $0.673 \pm 0.007$ & $0.507 \pm 0.096$ \\
\hline 0.58 & $0.622 \pm 0.005$ & $0.618 \pm 0.004$ & $0.481 \pm 0.003$ & $0.623 \pm 0.021$ & $0.674 \pm 0.011$ & $0.653 \pm 0.006$ & $0.533 \pm 0.020$ & $0.671 \pm 0.006$ & $0.495 \pm 0.108$ \\
\hline 0.57 & $0.619 \pm 0.004$ & $0.618 \pm 0.003$ & $0.483 \pm 0.002$ & $0.624 \pm 0.020$ & $0.676 \pm 0.010$ & $0.652 \pm 0.006$ & $0.531 \pm 0.018$ & $0.670 \pm 0.006$ & $0.501 \pm 0.105$ \\
\hline 0.56 & $0.617 \pm 0.003$ & $0.620 \pm 0.002$ & $0.484 \pm 0.001$ & $0.634 \pm 0.011$ & $0.673 \pm 0.007$ & $0.649 \pm 0.004$ & $0.527 \pm 0.015$ & $0.670 \pm 0.004$ & $0.497 \pm 0.105$ \\
\hline
\end{tabular}


Table S11. RMSE produced by all models on the CASF-2016 test set

\begin{tabular}{|c|c|c|c|c|c|c|c|c|c|}
\hline $\begin{array}{l}\text { Similarity } \\
\text { to test set }\end{array}$ & X-ScoreHM & ChemScore & ASP & L-SVR & $\mathrm{RF}$ & KNN & DT & BRR & MLP \\
\hline 0.73 & $1.80 \pm 0.00$ & $1.81 \pm 0.00$ & $1.90 \pm 0.00$ & $2.28 \pm 0.07$ & $1.38 \pm 0.00$ & $1.68 \pm 0.00$ & $1.64 \pm 0.01$ & $1.62 \pm 0.00$ & $3.76 \pm 0.96$ \\
\hline 0.72 & $1.79 \pm 0.01$ & $1.81 \pm 0.01$ & $1.90 \pm 0.00$ & $2.33 \pm 0.08$ & $1.39 \pm 0.01$ & $1.67 \pm 0.01$ & $1.65 \pm 0.02$ & $1.63 \pm 0.00$ & $3.55 \pm 0.75$ \\
\hline 0.71 & $1.79 \pm 0.01$ & $1.80 \pm 0.01$ & $1.91 \pm 0.01$ & $2.33 \pm 0.11$ & $1.41 \pm 0.02$ & $1.66 \pm 0.01$ & $1.66 \pm 0.03$ & $1.64 \pm 0.01$ & $3.67 \pm 0.82$ \\
\hline 0.70 & $1.78 \pm 0.01$ & $1.80 \pm 0.01$ & $1.92 \pm 0.01$ & $2.38 \pm 0.12$ & $1.44 \pm 0.02$ & $1.67 \pm 0.01$ & $1.67 \pm 0.03$ & $1.66 \pm 0.01$ & $3.90 \pm 1.08$ \\
\hline 0.69 & $1.76 \pm 0.01$ & $1.79 \pm 0.01$ & $1.93 \pm 0.01$ & $2.34 \pm 0.13$ & $1.45 \pm 0.02$ & $1.66 \pm 0.01$ & $1.67 \pm 0.03$ & $1.67 \pm 0.01$ & $3.66 \pm 0.95$ \\
\hline 0.68 & $1.75 \pm 0.01$ & $1.78 \pm 0.01$ & $1.93 \pm 0.01$ & $2.25 \pm 0.12$ & $1.46 \pm 0.02$ & $1.66 \pm 0.01$ & $1.69 \pm 0.03$ & $1.67 \pm 0.01$ & $3.72 \pm 0.98$ \\
\hline 0.67 & $1.75 \pm 0.01$ & $1.78 \pm 0.01$ & $1.94 \pm 0.01$ & $2.22 \pm 0.14$ & $1.47 \pm 0.02$ & $1.66 \pm 0.01$ & $1.70 \pm 0.03$ & $1.68 \pm 0.01$ & $3.89 \pm 0.91$ \\
\hline 0.66 & $1.75 \pm 0.01$ & $1.77 \pm 0.01$ & $1.94 \pm 0.01$ & $2.22 \pm 0.13$ & $1.49 \pm 0.02$ & $1.66 \pm 0.01$ & $1.71 \pm 0.03$ & $1.69 \pm 0.01$ & $3.87 \pm 1.00$ \\
\hline 0.65 & $1.75 \pm 0.01$ & $1.77 \pm 0.01$ & $1.94 \pm 0.01$ & $2.24 \pm 0.12$ & $1.50 \pm 0.03$ & $1.66 \pm 0.01$ & $1.73 \pm 0.03$ & $1.69 \pm 0.01$ & $3.77 \pm 1.01$ \\
\hline 0.64 & $1.75 \pm 0.01$ & $1.76 \pm 0.01$ & $1.94 \pm 0.01$ & $2.24 \pm 0.14$ & $1.51 \pm 0.03$ & $1.66 \pm 0.01$ & $1.74 \pm 0.03$ & $1.70 \pm 0.01$ & $3.79 \pm 0.99$ \\
\hline 0.63 & $1.75 \pm 0.01$ & $1.77 \pm 0.01$ & $1.95 \pm 0.01$ & $2.26 \pm 0.12$ & $1.53 \pm 0.03$ & $1.67 \pm 0.01$ & $1.76 \pm 0.03$ & $1.70 \pm 0.01$ & $3.90 \pm 0.90$ \\
\hline 0.62 & $1.76 \pm 0.01$ & $1.78 \pm 0.01$ & $1.96 \pm 0.01$ & $2.27 \pm 0.12$ & $1.56 \pm 0.03$ & $1.68 \pm 0.01$ & $1.78 \pm 0.03$ & $1.71 \pm 0.01$ & $4.13 \pm 1.10$ \\
\hline 0.61 & $1.76 \pm 0.01$ & $1.78 \pm 0.01$ & $1.97 \pm 0.01$ & $2.27 \pm 0.13$ & $1.57 \pm 0.02$ & $1.68 \pm 0.01$ & $1.79 \pm 0.03$ & $1.72 \pm 0.01$ & $3.99 \pm 0.93$ \\
\hline 0.60 & $1.76 \pm 0.01$ & $1.78 \pm 0.01$ & $1.99 \pm 0.02$ & $2.29 \pm 0.13$ & $1.60 \pm 0.03$ & $1.69 \pm 0.01$ & $1.80 \pm 0.03$ & $1.73 \pm 0.01$ & $4.14 \pm 1.07$ \\
\hline 0.59 & $1.78 \pm 0.01$ & $1.79 \pm 0.01$ & $2.02 \pm 0.02$ & $2.28 \pm 0.13$ & $1.64 \pm 0.03$ & $1.72 \pm 0.01$ & $1.84 \pm 0.04$ & $1.75 \pm 0.01$ & $4.11 \pm 1.06$ \\
\hline 0.58 & $1.79 \pm 0.01$ & $1.80 \pm 0.01$ & $2.03 \pm 0.02$ & $2.29 \pm 0.11$ & $1.66 \pm 0.02$ & $1.74 \pm 0.01$ & $1.86 \pm 0.03$ & $1.77 \pm 0.01$ & $4.15 \pm 0.99$ \\
\hline 0.57 & $1.80 \pm 0.01$ & $1.80 \pm 0.01$ & $2.04 \pm 0.02$ & $2.30 \pm 0.13$ & $1.65 \pm 0.02$ & $1.74 \pm 0.01$ & $1.86 \pm 0.03$ & $1.78 \pm 0.01$ & $4.12 \pm 0.94$ \\
\hline 0.56 & $1.80 \pm 0.00$ & $1.80 \pm 0.00$ & $2.04 \pm 0.01$ & $2.20 \pm 0.10$ & $1.66 \pm 0.01$ & $1.76 \pm 0.01$ & $1.87 \pm 0.02$ & $1.78 \pm 0.01$ & $4.28 \pm 1.03$ \\
\hline
\end{tabular}


Table S12. Spearman correlation coefficients produced by all models on the CASF-2016 test set

\begin{tabular}{|c|c|c|c|c|c|c|c|c|c|}
\hline $\begin{array}{l}\text { Similarity } \\
\text { to test set }\end{array}$ & X-Score ${ }^{\mathrm{HM}}$ & ChemScore & ASP & L-SVR & $\mathrm{RF}$ & KNN & DT & BRR & MLP \\
\hline 0.73 & $0.602 \pm 0.001$ & $0.575 \pm 0.001$ & $0.604 \pm 0.001$ & $0.679 \pm 0.007$ & $0.799 \pm 0.002$ & $0.681 \pm 0.001$ & $0.644 \pm 0.008$ & $0.712 \pm 0.001$ & $0.554 \pm 0.099$ \\
\hline 0.72 & $0.601 \pm 0.003$ & $0.575 \pm 0.003$ & $0.599 \pm 0.004$ & $0.668 \pm 0.015$ & $0.794 \pm 0.006$ & $0.679 \pm 0.003$ & $0.644 \pm 0.011$ & $0.705 \pm 0.002$ & $0.561 \pm 0.076$ \\
\hline 0.71 & $0.599 \pm 0.004$ & $0.573 \pm 0.005$ & $0.587 \pm 0.007$ & $0.668 \pm 0.021$ & $0.785 \pm 0.008$ & $0.679 \pm 0.004$ & $0.640 \pm 0.015$ & $0.703 \pm 0.003$ & $0.567 \pm 0.075$ \\
\hline 0.70 & $0.601 \pm 0.006$ & $0.575 \pm 0.005$ & $0.570 \pm 0.009$ & $0.647 \pm 0.023$ & $0.772 \pm 0.010$ & $0.677 \pm 0.005$ & $0.636 \pm 0.014$ & $0.696 \pm 0.004$ & $0.541 \pm 0.096$ \\
\hline 0.69 & $0.609 \pm 0.007$ & $0.584 \pm 0.008$ & $0.570 \pm 0.009$ & $0.652 \pm 0.020$ & $0.765 \pm 0.010$ & $0.677 \pm 0.005$ & $0.637 \pm 0.016$ & $0.695 \pm 0.004$ & $0.556 \pm 0.097$ \\
\hline 0.68 & $0.616 \pm 0.005$ & $0.591 \pm 0.007$ & $0.568 \pm 0.010$ & $0.671 \pm 0.022$ & $0.759 \pm 0.012$ & $0.676 \pm 0.005$ & $0.632 \pm 0.018$ & $0.694 \pm 0.005$ & $0.544 \pm 0.091$ \\
\hline 0.67 & $0.617 \pm 0.005$ & $0.595 \pm 0.006$ & $0.564 \pm 0.010$ & $0.674 \pm 0.020$ & $0.755 \pm 0.012$ & $0.675 \pm 0.005$ & $0.626 \pm 0.019$ & $0.691 \pm 0.006$ & $0.542 \pm 0.091$ \\
\hline 0.66 & $0.617 \pm 0.005$ & $0.602 \pm 0.006$ & $0.570 \pm 0.013$ & $0.666 \pm 0.022$ & $0.748 \pm 0.012$ & $0.676 \pm 0.005$ & $0.620 \pm 0.017$ & $0.690 \pm 0.006$ & $0.539 \pm 0.096$ \\
\hline 0.65 & $0.618 \pm 0.005$ & $0.607 \pm 0.006$ & $0.579 \pm 0.013$ & $0.660 \pm 0.020$ & $0.743 \pm 0.014$ & $0.676 \pm 0.007$ & $0.608 \pm 0.019$ & $0.688 \pm 0.006$ & $0.548 \pm 0.095$ \\
\hline 0.64 & $0.618 \pm 0.004$ & $0.609 \pm 0.006$ & $0.587 \pm 0.011$ & $0.665 \pm 0.019$ & $0.737 \pm 0.015$ & $0.677 \pm 0.006$ & $0.605 \pm 0.019$ & $0.687 \pm 0.006$ & $0.553 \pm 0.091$ \\
\hline 0.63 & $0.618 \pm 0.004$ & $0.608 \pm 0.006$ & $0.584 \pm 0.012$ & $0.663 \pm 0.017$ & $0.728 \pm 0.013$ & $0.678 \pm 0.006$ & $0.598 \pm 0.020$ & $0.688 \pm 0.006$ & $0.545 \pm 0.092$ \\
\hline 0.62 & $0.616 \pm 0.005$ & $0.609 \pm 0.006$ & $0.586 \pm 0.013$ & $0.663 \pm 0.017$ & $0.712 \pm 0.014$ & $0.678 \pm 0.006$ & $0.583 \pm 0.018$ & $0.688 \pm 0.006$ & $0.529 \pm 0.120$ \\
\hline 0.61 & $0.615 \pm 0.006$ & $0.613 \pm 0.005$ & $0.593 \pm 0.010$ & $0.660 \pm 0.022$ & $0.709 \pm 0.013$ & $0.677 \pm 0.007$ & $0.579 \pm 0.022$ & $0.690 \pm 0.005$ & $0.535 \pm 0.097$ \\
\hline 0.60 & $0.616 \pm 0.004$ & $0.614 \pm 0.004$ & $0.596 \pm 0.009$ & $0.661 \pm 0.019$ & $0.695 \pm 0.015$ & $0.674 \pm 0.007$ & $0.577 \pm 0.020$ & $0.691 \pm 0.005$ & $0.524 \pm 0.104$ \\
\hline 0.59 & $0.615 \pm 0.005$ & $0.615 \pm 0.004$ & $0.599 \pm 0.008$ & $0.662 \pm 0.014$ & $0.686 \pm 0.014$ & $0.663 \pm 0.007$ & $0.557 \pm 0.023$ & $0.689 \pm 0.006$ & $0.520 \pm 0.097$ \\
\hline 0.58 & $0.612 \pm 0.005$ & $0.613 \pm 0.004$ & $0.601 \pm 0.007$ & $0.660 \pm 0.015$ & $0.676 \pm 0.012$ & $0.657 \pm 0.007$ & $0.544 \pm 0.021$ & $0.687 \pm 0.005$ & $0.507 \pm 0.111$ \\
\hline 0.57 & $0.609 \pm 0.004$ & $0.612 \pm 0.003$ & $0.608 \pm 0.005$ & $0.661 \pm 0.016$ & $0.679 \pm 0.011$ & $0.654 \pm 0.007$ & $0.543 \pm 0.019$ & $0.685 \pm 0.005$ & $0.512 \pm 0.107$ \\
\hline 0.56 & $0.606 \pm 0.003$ & $0.611 \pm 0.002$ & $0.615 \pm 0.003$ & $0.671 \pm 0.008$ & $0.677 \pm 0.007$ & $0.650 \pm 0.004$ & $0.539 \pm 0.016$ & $0.687 \pm 0.003$ & $0.509 \pm 0.107$ \\
\hline
\end{tabular}


PART 5. Performance of all models calibrated on the training sets with different sizes (average similarity defined by protein sequence)

Table S13. Pearson correlation coefficients produced by all models on the CASF-2016 test set

\begin{tabular}{|c|c|c|c|c|c|c|c|c|c|}
\hline $\begin{array}{l}\text { Training } \\
\text { set size }\end{array}$ & $\mathrm{X}$-Score ${ }^{\mathrm{HM}}$ & ChemScore & ASP & L-SVR & $\mathrm{RF}$ & KNN & DT & BRR & MLP \\
\hline 3000 & $0.628 \pm 0.001$ & $0.605 \pm 0.001$ & $0.479 \pm 0.001$ & $0.655 \pm 0.004$ & $0.798 \pm 0.003$ & $0.699 \pm 0.001$ & $0.664 \pm 0.012$ & $0.668 \pm 0.001$ & $0.699 \pm 0.006$ \\
\hline 2750 & $0.628 \pm 0.002$ & $0.605 \pm 0.002$ & $0.479 \pm 0.002$ & $0.655 \pm 0.006$ & $0.794 \pm 0.005$ & $0.695 \pm 0.002$ & $0.658 \pm 0.014$ & $0.667 \pm 0.002$ & $0.695 \pm 0.008$ \\
\hline 2500 & $0.628 \pm 0.003$ & $0.605 \pm 0.003$ & $0.479 \pm 0.002$ & $0.653 \pm 0.006$ & $0.788 \pm 0.008$ & $0.689 \pm 0.003$ & $0.652 \pm 0.016$ & $0.667 \pm 0.004$ & $0.684 \pm 0.017$ \\
\hline 2250 & $0.628 \pm 0.003$ & $0.605 \pm 0.003$ & $0.479 \pm 0.002$ & $0.653 \pm 0.009$ & $0.784 \pm 0.008$ & $0.685 \pm 0.004$ & $0.647 \pm 0.016$ & $0.667 \pm 0.004$ & $0.668 \pm 0.032$ \\
\hline 2000 & $0.627 \pm 0.004$ & $0.605 \pm 0.004$ & $0.479 \pm 0.002$ & $0.651 \pm 0.010$ & $0.776 \pm 0.011$ & $0.680 \pm 0.004$ & $0.636 \pm 0.018$ & $0.667 \pm 0.005$ & $0.664 \pm 0.026$ \\
\hline 1750 & $0.628 \pm 0.004$ & $0.604 \pm 0.005$ & $0.479 \pm 0.004$ & $0.648 \pm 0.015$ & $0.770 \pm 0.010$ & $0.674 \pm 0.005$ & $0.631 \pm 0.018$ & $0.666 \pm 0.006$ & $0.654 \pm 0.028$ \\
\hline 1500 & $0.628 \pm 0.006$ & $0.605 \pm 0.005$ & $0.479 \pm 0.004$ & $0.647 \pm 0.012$ & $0.758 \pm 0.011$ & $0.671 \pm 0.006$ & $0.614 \pm 0.022$ & $0.665 \pm 0.006$ & $0.529 \pm 0.091$ \\
\hline 1250 & $0.627 \pm 0.006$ & $0.603 \pm 0.008$ & $0.478 \pm 0.005$ & $0.642 \pm 0.020$ & $0.749 \pm 0.014$ & $0.671 \pm 0.007$ & $0.598 \pm 0.023$ & $0.664 \pm 0.007$ & $0.426 \pm 0.118$ \\
\hline 1000 & $0.627 \pm 0.007$ & $0.604 \pm 0.008$ & $0.478 \pm 0.006$ & $0.637 \pm 0.025$ & $0.737 \pm 0.016$ & $0.670 \pm 0.008$ & $0.583 \pm 0.025$ & $0.663 \pm 0.009$ & $0.366 \pm 0.123$ \\
\hline 750 & $0.625 \pm 0.008$ & $0.601 \pm 0.009$ & $0.477 \pm 0.007$ & $0.624 \pm 0.040$ & $0.722 \pm 0.016$ & $0.667 \pm 0.009$ & $0.558 \pm 0.024$ & $0.662 \pm 0.010$ & $0.322 \pm 0.142$ \\
\hline
\end{tabular}

Table S14. RMSE produced by all models on the CASF-2016 test set

\begin{tabular}{|c|c|c|c|c|c|c|c|c|c|}
\hline $\begin{array}{l}\text { Training } \\
\text { set size }\end{array}$ & X-Score ${ }^{\mathrm{HM}}$ & ChemScore & ASP & L-SVR & $\mathrm{RF}$ & KNN & DT & BRR & MLP \\
\hline 3000 & $1.75 \pm 0.00$ & $1.76 \pm 0.00$ & $1.92 \pm 0.00$ & $2.19 \pm 0.09$ & $1.40 \pm 0.01$ & $1.61 \pm 0.00$ & $1.63 \pm 0.02$ & $1.68 \pm 0.00$ & $1.69 \pm 0.06$ \\
\hline 2750 & $1.75 \pm 0.00$ & $1.76 \pm 0.00$ & $1.92 \pm 0.00$ & $2.18 \pm 0.08$ & $1.41 \pm 0.01$ & $1.61 \pm 0.00$ & $1.64 \pm 0.03$ & $1.68 \pm 0.00$ & $1.72 \pm 0.07$ \\
\hline 2500 & $1.75 \pm 0.00$ & $1.76 \pm 0.01$ & $1.92 \pm 0.00$ & $2.18 \pm 0.10$ & $1.42 \pm 0.02$ & $1.63 \pm 0.01$ & $1.65 \pm 0.03$ & $1.68 \pm 0.01$ & $1.83 \pm 0.15$ \\
\hline 2250 & $1.75 \pm 0.01$ & $1.76 \pm 0.01$ & $1.92 \pm 0.00$ & $2.19 \pm 0.11$ & $1.43 \pm 0.02$ & $1.63 \pm 0.01$ & $1.66 \pm 0.03$ & $1.68 \pm 0.01$ & $2.06 \pm 0.31$ \\
\hline 2000 & $1.75 \pm 0.01$ & $1.76 \pm 0.01$ & $1.92 \pm 0.01$ & $2.21 \pm 0.11$ & $1.44 \pm 0.02$ & $1.64 \pm 0.01$ & $1.68 \pm 0.03$ & $1.68 \pm 0.01$ & $2.18 \pm 0.30$ \\
\hline 1750 & $1.75 \pm 0.01$ & $1.76 \pm 0.01$ & $1.93 \pm 0.01$ & $2.21 \pm 0.13$ & $1.46 \pm 0.02$ & $1.66 \pm 0.01$ & $1.69 \pm 0.03$ & $1.68 \pm 0.01$ & $2.30 \pm 0.28$ \\
\hline 1500 & $1.75 \pm 0.01$ & $1.76 \pm 0.01$ & $1.93 \pm 0.01$ & $2.21 \pm 0.12$ & $1.48 \pm 0.02$ & $1.67 \pm 0.01$ & $1.72 \pm 0.04$ & $1.68 \pm 0.01$ & $3.94 \pm 0.88$ \\
\hline 1250 & $1.75 \pm 0.01$ & $1.76 \pm 0.01$ & $1.93 \pm 0.01$ & $2.21 \pm 0.13$ & $1.50 \pm 0.03$ & $1.67 \pm 0.01$ & $1.75 \pm 0.04$ & $1.68 \pm 0.01$ & $5.08 \pm 1.27$ \\
\hline 1000 & $1.75 \pm 0.01$ & $1.76 \pm 0.01$ & $1.93 \pm 0.01$ & $2.25 \pm 0.15$ & $1.52 \pm 0.03$ & $1.68 \pm 0.02$ & $1.78 \pm 0.04$ & $1.68 \pm 0.01$ & $6.56 \pm 1.33$ \\
\hline 750 & $1.75 \pm 0.02$ & $1.77 \pm 0.02$ & $1.93 \pm 0.01$ & $2.31 \pm 0.19$ & $1.55 \pm 0.03$ & $1.70 \pm 0.02$ & $1.83 \pm 0.04$ & $1.69 \pm 0.02$ & $6.85 \pm 1.33$ \\
\hline
\end{tabular}


Table S15. Spearman correlation coefficients produced by all models on the CASF-2016 test set

\begin{tabular}{|c|c|c|c|c|c|c|c|c|c|}
\hline $\begin{array}{l}\text { Training } \\
\text { set size }\end{array}$ & X-Score ${ }^{H M}$ & ChemScore & ASP & L-SVR & RF & KNN & DT & BRR & MLP \\
\hline 3000 & $0.618 \pm 0.001$ & $0.604 \pm 0.001$ & $0.587 \pm 0.002$ & $0.676 \pm 0.004$ & $0.793 \pm 0.003$ & $0.702 \pm 0.002$ & $0.667 \pm 0.010$ & $0.695 \pm 0.001$ & $0.708 \pm 0.005$ \\
\hline 2750 & $0.617 \pm 0.002$ & $0.604 \pm 0.002$ & $0.588 \pm 0.004$ & $0.676 \pm 0.006$ & $0.788 \pm 0.005$ & $0.697 \pm 0.003$ & $0.662 \pm 0.013$ & $0.695 \pm 0.002$ & $0.704 \pm 0.007$ \\
\hline 2500 & $0.617 \pm 0.003$ & $0.603 \pm 0.004$ & $0.587 \pm 0.005$ & $0.675 \pm 0.007$ & $0.782 \pm 0.008$ & $0.688 \pm 0.004$ & $0.656 \pm 0.016$ & $0.694 \pm 0.003$ & $0.695 \pm 0.017$ \\
\hline 2250 & $0.617 \pm 0.003$ & $0.604 \pm 0.004$ & $0.588 \pm 0.006$ & $0.675 \pm 0.009$ & $0.779 \pm 0.008$ & $0.684 \pm 0.005$ & $0.652 \pm 0.016$ & $0.695 \pm 0.003$ & $0.681 \pm 0.034$ \\
\hline 2000 & $0.617 \pm 0.004$ & $0.604 \pm 0.005$ & $0.588 \pm 0.006$ & $0.673 \pm 0.010$ & $0.770 \pm 0.011$ & $0.682 \pm 0.005$ & $0.639 \pm 0.018$ & $0.694 \pm 0.004$ & $0.678 \pm 0.026$ \\
\hline 1750 & $0.617 \pm 0.004$ & $0.603 \pm 0.006$ & $0.586 \pm 0.009$ & $0.670 \pm 0.015$ & $0.764 \pm 0.010$ & $0.677 \pm 0.006$ & $0.633 \pm 0.017$ & $0.694 \pm 0.004$ & $0.670 \pm 0.028$ \\
\hline 1500 & $0.617 \pm 0.005$ & $0.603 \pm 0.005$ & $0.587 \pm 0.010$ & $0.669 \pm 0.013$ & $0.755 \pm 0.011$ & $0.675 \pm 0.007$ & $0.617 \pm 0.022$ & $0.693 \pm 0.005$ & $0.542 \pm 0.094$ \\
\hline 1250 & $0.617 \pm 0.005$ & $0.602 \pm 0.008$ & $0.586 \pm 0.012$ & $0.665 \pm 0.019$ & $0.744 \pm 0.014$ & $0.673 \pm 0.006$ & $0.600 \pm 0.024$ & $0.692 \pm 0.006$ & $0.437 \pm 0.121$ \\
\hline 1000 & $0.616 \pm 0.007$ & $0.603 \pm 0.007$ & $0.587 \pm 0.014$ & $0.661 \pm 0.025$ & $0.732 \pm 0.017$ & $0.676 \pm 0.007$ & $0.583 \pm 0.026$ & $0.691 \pm 0.007$ & $0.376 \pm 0.126$ \\
\hline 750 & $0.615 \pm 0.008$ & $0.601 \pm 0.008$ & $0.584 \pm 0.017$ & $0.648 \pm 0.041$ & $0.719 \pm 0.017$ & $0.676 \pm 0.008$ & $0.558 \pm 0.027$ & $0.689 \pm 0.009$ & $0.330 \pm 0.144$ \\
\hline
\end{tabular}


PART 6. Performance of all models calibrated on the training sets with different sizes (average similarity defined by ligand shape)

Table S16. Pearson correlation coefficients produced by all models on the CASF-2016 test set

\begin{tabular}{|c|c|c|c|c|c|c|c|c|c|}
\hline $\begin{array}{l}\text { Training } \\
\text { set size }\end{array}$ & X-Score ${ }^{H M}$ & ChemScore & ASP & L-SVR & RF & KNN & DT & BRR & MLP \\
\hline 3000 & $0.632 \pm 0.002$ & $0.606 \pm 0.002$ & $0.482 \pm 0.002$ & $0.655 \pm 0.008$ & $0.782 \pm 0.006$ & $0.700 \pm 0.003$ & $0.648 \pm 0.011$ & $0.658 \pm 0.003$ & $0.690 \pm 0.008$ \\
\hline 2750 & $0.631 \pm 0.002$ & $0.607 \pm 0.003$ & $0.482 \pm 0.002$ & $0.654 \pm 0.010$ & $0.778 \pm 0.006$ & $0.695 \pm 0.003$ & $0.638 \pm 0.013$ & $0.658 \pm 0.004$ & $0.688 \pm 0.008$ \\
\hline 2500 & $0.632 \pm 0.003$ & $0.607 \pm 0.004$ & $0.482 \pm 0.002$ & $0.652 \pm 0.011$ & $0.773 \pm 0.008$ & $0.691 \pm 0.004$ & $0.634 \pm 0.014$ & $0.658 \pm 0.005$ & $0.680 \pm 0.014$ \\
\hline 2250 & $0.632 \pm 0.003$ & $0.607 \pm 0.004$ & $0.481 \pm 0.003$ & $0.648 \pm 0.014$ & $0.768 \pm 0.009$ & $0.689 \pm 0.004$ & $0.629 \pm 0.015$ & $0.658 \pm 0.005$ & $0.663 \pm 0.035$ \\
\hline 2000 & $0.632 \pm 0.003$ & $0.607 \pm 0.004$ & $0.482 \pm 0.003$ & $0.645 \pm 0.017$ & $0.761 \pm 0.010$ & $0.684 \pm 0.004$ & $0.619 \pm 0.018$ & $0.658 \pm 0.006$ & $0.654 \pm 0.036$ \\
\hline 1750 & $0.632 \pm 0.004$ & $0.606 \pm 0.005$ & $0.481 \pm 0.004$ & $0.640 \pm 0.018$ & $0.755 \pm 0.010$ & $0.680 \pm 0.004$ & $0.612 \pm 0.018$ & $0.657 \pm 0.008$ & $0.646 \pm 0.040$ \\
\hline 1500 & $0.631 \pm 0.005$ & $0.606 \pm 0.005$ & $0.481 \pm 0.004$ & $0.636 \pm 0.021$ & $0.746 \pm 0.012$ & $0.674 \pm 0.005$ & $0.603 \pm 0.021$ & $0.660 \pm 0.009$ & $0.524 \pm 0.093$ \\
\hline 1250 & $0.630 \pm 0.006$ & $0.604 \pm 0.008$ & $0.481 \pm 0.005$ & $0.630 \pm 0.025$ & $0.735 \pm 0.011$ & $0.673 \pm 0.007$ & $0.584 \pm 0.021$ & $0.657 \pm 0.010$ & $0.438 \pm 0.110$ \\
\hline 1000 & $0.630 \pm 0.007$ & $0.605 \pm 0.008$ & $0.480 \pm 0.004$ & $0.625 \pm 0.027$ & $0.722 \pm 0.017$ & $0.670 \pm 0.008$ & $0.565 \pm 0.024$ & $0.656 \pm 0.010$ & $0.367 \pm 0.130$ \\
\hline 750 & $0.629 \pm 0.009$ & $0.602 \pm 0.012$ & $0.479 \pm 0.007$ & $0.609 \pm 0.038$ & $0.704 \pm 0.017$ & $0.664 \pm 0.009$ & $0.537 \pm 0.027$ & $0.656 \pm 0.013$ & $0.330 \pm 0.131$ \\
\hline
\end{tabular}

Table S17. RMSE produced by all models on the CASF-2016 test set

\begin{tabular}{|c|c|c|c|c|c|c|c|c|c|}
\hline $\begin{array}{l}\text { Training } \\
\text { set size }\end{array}$ & X-Score ${ }^{\mathrm{HM}}$ & ChemScore & ASP & L-SVR & $\mathrm{RF}$ & KNN & DT & BRR & MLP \\
\hline 3000 & $1.74 \pm 0.00$ & $1.77 \pm 0.00$ & $1.94 \pm 0.01$ & $2.17 \pm 0.09$ & $1.44 \pm 0.01$ & $1.61 \pm 0.01$ & $1.66 \pm 0.02$ & $1.70 \pm 0.01$ & $1.70 \pm 0.06$ \\
\hline 2750 & $1.74 \pm 0.00$ & $1.76 \pm 0.00$ & $1.94 \pm 0.01$ & $2.17 \pm 0.08$ & $1.45 \pm 0.01$ & $1.62 \pm 0.01$ & $1.68 \pm 0.02$ & $1.70 \pm 0.01$ & $1.74 \pm 0.08$ \\
\hline 2500 & $1.74 \pm 0.01$ & $1.77 \pm 0.01$ & $1.94 \pm 0.01$ & $2.18 \pm 0.10$ & $1.46 \pm 0.02$ & $1.63 \pm 0.01$ & $1.69 \pm 0.02$ & $1.70 \pm 0.01$ & $1.82 \pm 0.12$ \\
\hline 2250 & $1.74 \pm 0.01$ & $1.77 \pm 0.01$ & $1.94 \pm 0.01$ & $2.19 \pm 0.09$ & $1.47 \pm 0.02$ & $1.63 \pm 0.01$ & $1.69 \pm 0.03$ & $1.70 \pm 0.01$ & $2.00 \pm 0.25$ \\
\hline 2000 & $1.74 \pm 0.01$ & $1.76 \pm 0.01$ & $1.94 \pm 0.01$ & $2.21 \pm 0.11$ & $1.48 \pm 0.02$ & $1.64 \pm 0.01$ & $1.71 \pm 0.03$ & $1.70 \pm 0.01$ & $2.24 \pm 0.36$ \\
\hline 1750 & $1.74 \pm 0.01$ & $1.77 \pm 0.01$ & $1.94 \pm 0.01$ & $2.25 \pm 0.15$ & $1.49 \pm 0.02$ & $1.65 \pm 0.01$ & $1.72 \pm 0.03$ & $1.70 \pm 0.01$ & $2.36 \pm 0.36$ \\
\hline 1500 & $1.74 \pm 0.01$ & $1.77 \pm 0.01$ & $1.93 \pm 0.01$ & $2.28 \pm 0.12$ & $1.51 \pm 0.02$ & $1.66 \pm 0.01$ & $1.74 \pm 0.04$ & $1.70 \pm 0.01$ & $3.92 \pm 1.06$ \\
\hline 1250 & $1.75 \pm 0.01$ & $1.77 \pm 0.01$ & $1.94 \pm 0.01$ & $2.28 \pm 0.14$ & $1.53 \pm 0.02$ & $1.67 \pm 0.01$ & $1.77 \pm 0.04$ & $1.71 \pm 0.02$ & $4.90 \pm 1.02$ \\
\hline 1000 & $1.74 \pm 0.01$ & $1.77 \pm 0.02$ & $1.94 \pm 0.01$ & $2.33 \pm 0.16$ & $1.55 \pm 0.03$ & $1.68 \pm 0.02$ & $1.81 \pm 0.04$ & $1.71 \pm 0.02$ & $6.43 \pm 1.36$ \\
\hline 750 & $1.75 \pm 0.02$ & $1.77 \pm 0.02$ & $1.94 \pm 0.02$ & $2.37 \pm 0.19$ & $1.59 \pm 0.03$ & $1.70 \pm 0.02$ & $1.87 \pm 0.05$ & $1.71 \pm 0.02$ & $6.63 \pm 1.24$ \\
\hline
\end{tabular}


Table S18. Spearman correlation coefficients produced by all models on the CASF-2016 test set

\begin{tabular}{|c|c|c|c|c|c|c|c|c|c|}
\hline $\begin{array}{l}\text { Training } \\
\text { set size }\end{array}$ & X-Score ${ }^{H M}$ & ChemScore & ASP & L-SVR & $\mathrm{RF}$ & KNN & DT & BRR & MLP \\
\hline 3000 & $0.620 \pm 0.002$ & $0.609 \pm 0.002$ & $0.595 \pm 0.006$ & $0.685 \pm 0.006$ & $0.776 \pm 0.007$ & $0.698 \pm 0.003$ & $0.653 \pm 0.011$ & $0.686 \pm 0.002$ & $0.698 \pm 0.008$ \\
\hline 2750 & $0.620 \pm 0.003$ & $0.609 \pm 0.003$ & $0.596 \pm 0.005$ & $0.683 \pm 0.007$ & $0.772 \pm 0.007$ & $0.694 \pm 0.004$ & $0.642 \pm 0.012$ & $0.685 \pm 0.003$ & $0.696 \pm 0.007$ \\
\hline 2500 & $0.621 \pm 0.003$ & $0.609 \pm 0.004$ & $0.597 \pm 0.006$ & $0.681 \pm 0.009$ & $0.765 \pm 0.009$ & $0.690 \pm 0.005$ & $0.638 \pm 0.014$ & $0.686 \pm 0.004$ & $0.689 \pm 0.014$ \\
\hline 2250 & $0.621 \pm 0.004$ & $0.609 \pm 0.004$ & $0.595 \pm 0.008$ & $0.677 \pm 0.012$ & $0.761 \pm 0.010$ & $0.688 \pm 0.005$ & $0.635 \pm 0.015$ & $0.685 \pm 0.004$ & $0.676 \pm 0.034$ \\
\hline 2000 & $0.620 \pm 0.004$ & $0.609 \pm 0.004$ & $0.596 \pm 0.007$ & $0.674 \pm 0.015$ & $0.755 \pm 0.010$ & $0.685 \pm 0.005$ & $0.623 \pm 0.018$ & $0.685 \pm 0.005$ & $0.668 \pm 0.037$ \\
\hline 1750 & $0.621 \pm 0.005$ & $0.608 \pm 0.004$ & $0.594 \pm 0.009$ & $0.668 \pm 0.017$ & $0.749 \pm 0.011$ & $0.682 \pm 0.005$ & $0.615 \pm 0.018$ & $0.684 \pm 0.006$ & $0.661 \pm 0.040$ \\
\hline 1500 & $0.620 \pm 0.005$ & $0.608 \pm 0.006$ & $0.594 \pm 0.010$ & $0.666 \pm 0.017$ & $0.739 \pm 0.012$ & $0.677 \pm 0.005$ & $0.605 \pm 0.021$ & $0.686 \pm 0.007$ & $0.538 \pm 0.096$ \\
\hline 1250 & $0.619 \pm 0.006$ & $0.607 \pm 0.007$ & $0.595 \pm 0.013$ & $0.659 \pm 0.022$ & $0.730 \pm 0.013$ & $0.677 \pm 0.007$ & $0.587 \pm 0.023$ & $0.684 \pm 0.008$ & $0.449 \pm 0.113$ \\
\hline 1000 & $0.620 \pm 0.007$ & $0.606 \pm 0.007$ & $0.592 \pm 0.012$ & $0.652 \pm 0.027$ & $0.717 \pm 0.019$ & $0.678 \pm 0.007$ & $0.565 \pm 0.026$ & $0.683 \pm 0.009$ & $0.378 \pm 0.132$ \\
\hline 750 & $0.619 \pm 0.009$ & $0.605 \pm 0.011$ & $0.592 \pm 0.019$ & $0.636 \pm 0.03$ & $0.700 \pm 0.019$ & $0.675 \pm 0.007$ & $0.536 \pm 0.029$ & $0.682 \pm 0.010$ & $0.338 \pm 0.134$ \\
\hline
\end{tabular}


PART 7. Performance of all models calibrated on the training sets with different sizes (average similarity defined by binding pocket)

Table S19. Pearson correlation coefficients produced by all models on the CASF-2016 test set

\begin{tabular}{|c|c|c|c|c|c|c|c|c|c|}
\hline $\begin{array}{l}\text { Training } \\
\text { set size }\end{array}$ & X-Score ${ }^{H M}$ & ChemScore & ASP & L-SVR & RF & KNN & DT & BRR & MLP \\
\hline 3000 & $0.629 \pm 0.002$ & $0.607 \pm 0.002$ & $0.478 \pm 0.001$ & $0.645 \pm 0.008$ & $0.792 \pm 0.004$ & $0.705 \pm 0.002$ & $0.662 \pm 0.012$ & $0.665 \pm 0.009$ & $0.695 \pm 0.008$ \\
\hline 2750 & $0.629 \pm 0.002$ & $0.606 \pm 0.003$ & $0.478 \pm 0.002$ & $0.641 \pm 0.011$ & $0.788 \pm 0.005$ & $0.700 \pm 0.003$ & $0.655 \pm 0.013$ & $0.664 \pm 0.008$ & $0.693 \pm 0.008$ \\
\hline 2500 & $0.629 \pm 0.002$ & $0.606 \pm 0.003$ & $0.478 \pm 0.002$ & $0.640 \pm 0.012$ & $0.784 \pm 0.007$ & $0.696 \pm 0.004$ & $0.650 \pm 0.016$ & $0.665 \pm 0.007$ & $0.686 \pm 0.016$ \\
\hline 2250 & $0.629 \pm 0.003$ & $0.606 \pm 0.004$ & $0.477 \pm 0.003$ & $0.639 \pm 0.015$ & $0.778 \pm 0.007$ & $0.693 \pm 0.004$ & $0.644 \pm 0.013$ & $0.665 \pm 0.007$ & $0.670 \pm 0.026$ \\
\hline 2000 & $0.628 \pm 0.004$ & $0.605 \pm 0.004$ & $0.477 \pm 0.003$ & $0.638 \pm 0.018$ & $0.772 \pm 0.009$ & $0.688 \pm 0.004$ & $0.638 \pm 0.016$ & $0.666 \pm 0.005$ & $0.659 \pm 0.032$ \\
\hline 1750 & $0.628 \pm 0.004$ & $0.606 \pm 0.005$ & $0.477 \pm 0.003$ & $0.635 \pm 0.020$ & $0.761 \pm 0.012$ & $0.683 \pm 0.005$ & $0.623 \pm 0.019$ & $0.666 \pm 0.006$ & $0.655 \pm 0.027$ \\
\hline 1500 & $0.629 \pm 0.005$ & $0.606 \pm 0.006$ & $0.478 \pm 0.004$ & $0.626 \pm 0.022$ & $0.755 \pm 0.012$ & $0.679 \pm 0.005$ & $0.613 \pm 0.019$ & $0.667 \pm 0.004$ & $0.524 \pm 0.081$ \\
\hline 1250 & $0.629 \pm 0.006$ & $0.605 \pm 0.007$ & $0.476 \pm 0.005$ & $0.618 \pm 0.028$ & $0.744 \pm 0.014$ & $0.677 \pm 0.006$ & $0.597 \pm 0.023$ & $0.667 \pm 0.003$ & $0.426 \pm 0.115$ \\
\hline 1000 & $0.628 \pm 0.006$ & $0.605 \pm 0.009$ & $0.476 \pm 0.006$ & $0.619 \pm 0.034$ & $0.736 \pm 0.016$ & $0.674 \pm 0.007$ & $0.581 \pm 0.023$ & $0.667 \pm 0.002$ & $0.376 \pm 0.117$ \\
\hline 750 & $0.628 \pm 0.007$ & $0.604 \pm 0.010$ & $0.476 \pm 0.007$ & $0.602 \pm 0.040$ & $0.720 \pm 0.018$ & $0.670 \pm 0.009$ & $0.555 \pm 0.025$ & $0.665 \pm 0.011$ & $0.324 \pm 0.127$ \\
\hline
\end{tabular}

Table S20. RMSE produced by all models on the CASF-2016 test set

\begin{tabular}{|c|c|c|c|c|c|c|c|c|c|}
\hline $\begin{array}{l}\text { Training } \\
\text { set size }\end{array}$ & X-Score ${ }^{\mathrm{HM}}$ & ChemScore & ASP & L-SVR & $\mathrm{RF}$ & KNN & DT & BRR & MLP \\
\hline 3000 & $1.75 \pm 0.00$ & $1.76 \pm 0.00$ & $1.93 \pm 0.00$ & $2.18 \pm 0.08$ & $1.41 \pm 0.01$ & $1.60 \pm 0.00$ & $1.64 \pm 0.02$ & $1.69 \pm 0.02$ & $1.70 \pm 0.06$ \\
\hline 2750 & $1.75 \pm 0.00$ & $1.76 \pm 0.00$ & $1.93 \pm 0.00$ & $2.19 \pm 0.08$ & $1.42 \pm 0.01$ & $1.61 \pm 0.01$ & $1.65 \pm 0.02$ & $1.69 \pm 0.01$ & $1.74 \pm 0.07$ \\
\hline 2500 & $1.75 \pm 0.01$ & $1.76 \pm 0.01$ & $1.93 \pm 0.00$ & $2.20 \pm 0.09$ & $1.43 \pm 0.02$ & $1.62 \pm 0.01$ & $1.66 \pm 0.03$ & $1.68 \pm 0.01$ & $1.81 \pm 0.12$ \\
\hline 2250 & $1.75 \pm 0.01$ & $1.76 \pm 0.01$ & $1.94 \pm 0.01$ & $2.21 \pm 0.10$ & $1.44 \pm 0.02$ & $1.63 \pm 0.01$ & $1.67 \pm 0.02$ & $1.69 \pm 0.01$ & $2.01 \pm 0.23$ \\
\hline 2000 & $1.75 \pm 0.01$ & $1.76 \pm 0.01$ & $1.94 \pm 0.01$ & $2.22 \pm 0.10$ & $1.45 \pm 0.02$ & $1.64 \pm 0.01$ & $1.68 \pm 0.03$ & $1.68 \pm 0.01$ & $2.22 \pm 0.36$ \\
\hline 1750 & $1.75 \pm 0.01$ & $1.76 \pm 0.01$ & $1.94 \pm 0.01$ & $2.21 \pm 0.11$ & $1.47 \pm 0.03$ & $1.65 \pm 0.01$ & $1.70 \pm 0.03$ & $1.68 \pm 0.01$ & $2.32 \pm 0.32$ \\
\hline 1500 & $1.74 \pm 0.01$ & $1.76 \pm 0.01$ & $1.93 \pm 0.01$ & $2.26 \pm 0.12$ & $1.48 \pm 0.02$ & $1.66 \pm 0.01$ & $1.72 \pm 0.04$ & $1.68 \pm 0.01$ & $3.96 \pm 0.95$ \\
\hline 1250 & $1.75 \pm 0.01$ & $1.77 \pm 0.01$ & $1.94 \pm 0.01$ & $2.28 \pm 0.12$ & $1.51 \pm 0.03$ & $1.67 \pm 0.01$ & $1.75 \pm 0.04$ & $1.68 \pm 0.01$ & $5.15 \pm 1.20$ \\
\hline 1000 & $1.75 \pm 0.01$ & $1.76 \pm 0.01$ & $1.94 \pm 0.01$ & $2.30 \pm 0.14$ & $1.52 \pm 0.03$ & $1.68 \pm 0.02$ & $1.79 \pm 0.04$ & $1.68 \pm 0.00$ & $6.51 \pm 1.27$ \\
\hline 750 & $1.75 \pm 0.01$ & $1.77 \pm 0.02$ & $1.94 \pm 0.01$ & $2.36 \pm 0.18$ & $1.55 \pm 0.03$ & $1.69 \pm 0.02$ & $1.84 \pm 0.05$ & $1.69 \pm 0.02$ & $7.10 \pm 1.20$ \\
\hline
\end{tabular}


Table S21. Spearman correlation coefficients produced by all models on the CASF-2016 test set

\begin{tabular}{|c|c|c|c|c|c|c|c|c|c|}
\hline $\begin{array}{l}\text { Training } \\
\text { set size }\end{array}$ & X-Score ${ }^{H M}$ & ChemScore & ASP & L-SVR & RF & KNN & DT & BRR & MLP \\
\hline 3000 & $0.618 \pm 0.002$ & $0.609 \pm 0.002$ & $0.585 \pm 0.004$ & $0.680 \pm 0.006$ & $0.789 \pm 0.005$ & $0.706 \pm 0.003$ & $0.665 \pm 0.011$ & $0.690 \pm 0.007$ & $0.707 \pm 0.006$ \\
\hline 2750 & $0.618 \pm 0.002$ & $0.608 \pm 0.003$ & $0.584 \pm 0.005$ & $0.676 \pm 0.010$ & $0.784 \pm 0.006$ & $0.701 \pm 0.003$ & $0.659 \pm 0.013$ & $0.690 \pm 0.006$ & $0.705 \pm 0.006$ \\
\hline 2500 & $0.618 \pm 0.002$ & $0.609 \pm 0.003$ & $0.585 \pm 0.005$ & $0.675 \pm 0.011$ & $0.780 \pm 0.008$ & $0.696 \pm 0.004$ & $0.653 \pm 0.015$ & $0.691 \pm 0.005$ & $0.699 \pm 0.016$ \\
\hline 2250 & $0.617 \pm 0.003$ & $0.608 \pm 0.004$ & $0.583 \pm 0.007$ & $0.674 \pm 0.012$ & $0.773 \pm 0.008$ & $0.692 \pm 0.005$ & $0.648 \pm 0.014$ & $0.690 \pm 0.005$ & $0.685 \pm 0.027$ \\
\hline 2000 & $0.617 \pm 0.004$ & $0.607 \pm 0.004$ & $0.583 \pm 0.009$ & $0.672 \pm 0.014$ & $0.768 \pm 0.011$ & $0.687 \pm 0.005$ & $0.641 \pm 0.015$ & $0.691 \pm 0.004$ & $0.675 \pm 0.033$ \\
\hline 1750 & $0.617 \pm 0.004$ & $0.608 \pm 0.005$ & $0.583 \pm 0.009$ & $0.668 \pm 0.018$ & $0.756 \pm 0.012$ & $0.682 \pm 0.006$ & $0.626 \pm 0.019$ & $0.692 \pm 0.004$ & $0.672 \pm 0.027$ \\
\hline 1500 & $0.618 \pm 0.005$ & $0.607 \pm 0.006$ & $0.585 \pm 0.010$ & $0.660 \pm 0.020$ & $0.751 \pm 0.014$ & $0.680 \pm 0.006$ & $0.616 \pm 0.020$ & $0.692 \pm 0.003$ & $0.538 \pm 0.082$ \\
\hline 1250 & $0.618 \pm 0.006$ & $0.606 \pm 0.007$ & $0.583 \pm 0.012$ & $0.650 \pm 0.026$ & $0.739 \pm 0.016$ & $0.678 \pm 0.006$ & $0.600 \pm 0.024$ & $0.692 \pm 0.002$ & $0.437 \pm 0.117$ \\
\hline 1000 & $0.617 \pm 0.006$ & $0.606 \pm 0.009$ & $0.582 \pm 0.015$ & $0.651 \pm 0.032$ & $0.732 \pm 0.016$ & $0.678 \pm 0.007$ & $0.582 \pm 0.023$ & $0.692 \pm 0.002$ & $0.386 \pm 0.119$ \\
\hline 750 & $0.617 \pm 0.007$ & $0.606 \pm 0.010$ & $0.583 \pm 0.017$ & $0.633 \pm 0.039$ & $0.717 \pm 0.019$ & $0.677 \pm 0.007$ & $0.556 \pm 0.026$ & $0.689 \pm 0.009$ & $0.333 \pm 0.129$ \\
\hline
\end{tabular}


PART 8. Performance of all models calibrated on the training sets with different sizes (average similarity defined by the 3-in-1 combined metric)

Table S22. Pearson correlation coefficients produced by all models on the CASF-2016 test set

\begin{tabular}{|c|c|c|c|c|c|c|c|c|c|}
\hline $\begin{array}{c}\text { Training } \\
\text { set size }\end{array}$ & X-Score ${ }^{H M}$ & ChemScore & ASP & L-SVR & RF & KNN & DT & BRR & MLP \\
\hline 3000 & $0.630 \pm 0.002$ & $0.605 \pm 0.003$ & $0.471 \pm 0.002$ & $0.658 \pm 0.006$ & $0.796 \pm 0.005$ & $0.701 \pm 0.002$ & $0.672 \pm 0.012$ & $0.668 \pm 0.002$ & $0.695 \pm 0.007$ \\
\hline 2750 & $0.630 \pm 0.002$ & $0.605 \pm 0.003$ & $0.472 \pm 0.003$ & $0.655 \pm 0.010$ & $0.790 \pm 0.006$ & $0.697 \pm 0.003$ & $0.664 \pm 0.013$ & $0.667 \pm 0.003$ & $0.691 \pm 0.008$ \\
\hline 2500 & $0.630 \pm 0.002$ & $0.605 \pm 0.003$ & $0.471 \pm 0.003$ & $0.654 \pm 0.012$ & $0.785 \pm 0.007$ & $0.692 \pm 0.003$ & $0.658 \pm 0.014$ & $0.666 \pm 0.004$ & $0.680 \pm 0.015$ \\
\hline 2250 & $0.629 \pm 0.003$ & $0.604 \pm 0.004$ & $0.470 \pm 0.004$ & $0.650 \pm 0.014$ & $0.780 \pm 0.008$ & $0.687 \pm 0.004$ & $0.653 \pm 0.015$ & $0.667 \pm 0.004$ & $0.666 \pm 0.028$ \\
\hline 2000 & $0.630 \pm 0.004$ & $0.605 \pm 0.004$ & $0.471 \pm 0.004$ & $0.647 \pm 0.019$ & $0.772 \pm 0.010$ & $0.682 \pm 0.005$ & $0.640 \pm 0.017$ & $0.665 \pm 0.006$ & $0.657 \pm 0.039$ \\
\hline 1750 & $0.629 \pm 0.005$ & $0.604 \pm 0.005$ & $0.470 \pm 0.005$ & $0.644 \pm 0.022$ & $0.763 \pm 0.012$ & $0.677 \pm 0.005$ & $0.629 \pm 0.019$ & $0.665 \pm 0.006$ & $0.652 \pm 0.028$ \\
\hline 1500 & $0.628 \pm 0.005$ & $0.603 \pm 0.006$ & $0.471 \pm 0.006$ & $0.639 \pm 0.024$ & $0.754 \pm 0.011$ & $0.673 \pm 0.006$ & $0.618 \pm 0.017$ & $0.665 \pm 0.008$ & $0.525 \pm 0.094$ \\
\hline 1250 & $0.628 \pm 0.006$ & $0.602 \pm 0.008$ & $0.470 \pm 0.006$ & $0.628 \pm 0.032$ & $0.746 \pm 0.013$ & $0.672 \pm 0.006$ & $0.605 \pm 0.022$ & $0.664 \pm 0.009$ & $0.431 \pm 0.098$ \\
\hline 1000 & $0.628 \pm 0.007$ & $0.603 \pm 0.008$ & $0.470 \pm 0.007$ & $0.622 \pm 0.035$ & $0.733 \pm 0.016$ & $0.670 \pm 0.008$ & $0.583 \pm 0.021$ & $0.664 \pm 0.009$ & $0.355 \pm 0.114$ \\
\hline 750 & $0.625 \pm 0.009$ & $0.599 \pm 0.010$ & $0.467 \pm 0.010$ & $0.616 \pm 0.042$ & $0.717 \pm 0.018$ & $0.665 \pm 0.009$ & $0.558 \pm 0.027$ & $0.661 \pm 0.013$ & $0.308 \pm 0.125$ \\
\hline
\end{tabular}

Table S23. RMSE produced by all models on the CASF-2016 test set

\begin{tabular}{|c|c|c|c|c|c|c|c|c|c|}
\hline $\begin{array}{l}\text { Training } \\
\text { set size }\end{array}$ & $\mathrm{X}$-Score ${ }^{\mathrm{HM}}$ & ChemScore & ASP & L-SVR & $\mathrm{RF}$ & KNN & DT & BRR & MLP \\
\hline 3000 & $1.75 \pm 0.00$ & $1.77 \pm 0.00$ & $1.93 \pm 0.00$ & $2.12 \pm 0.08$ & $1.40 \pm 0.01$ & $1.60 \pm 0.00$ & $1.62 \pm 0.02$ & $1.68 \pm 0.00$ & $1.71 \pm 0.06$ \\
\hline 2750 & $1.75 \pm 0.00$ & $1.77 \pm 0.01$ & $1.93 \pm 0.00$ & $2.13 \pm 0.09$ & $1.41 \pm 0.02$ & $1.61 \pm 0.01$ & $1.63 \pm 0.02$ & $1.68 \pm 0.01$ & $1.74 \pm 0.07$ \\
\hline 2500 & $1.75 \pm 0.01$ & $1.77 \pm 0.01$ & $1.94 \pm 0.00$ & $2.13 \pm 0.10$ & $1.42 \pm 0.02$ & $1.62 \pm 0.01$ & $1.64 \pm 0.03$ & $1.68 \pm 0.01$ & $1.85 \pm 0.14$ \\
\hline 2250 & $1.75 \pm 0.01$ & $1.77 \pm 0.01$ & $1.94 \pm 0.01$ & $2.16 \pm 0.10$ & $1.43 \pm 0.02$ & $1.63 \pm 0.01$ & $1.65 \pm 0.03$ & $1.68 \pm 0.01$ & $2.04 \pm 0.25$ \\
\hline 2000 & $1.75 \pm 0.01$ & $1.77 \pm 0.01$ & $1.94 \pm 0.01$ & $2.18 \pm 0.12$ & $1.45 \pm 0.02$ & $1.64 \pm 0.01$ & $1.67 \pm 0.03$ & $1.69 \pm 0.01$ & $2.23 \pm 0.37$ \\
\hline 1750 & $1.75 \pm 0.01$ & $1.77 \pm 0.01$ & $1.94 \pm 0.01$ & $2.20 \pm 0.14$ & $1.47 \pm 0.02$ & $1.65 \pm 0.01$ & $1.69 \pm 0.03$ & $1.69 \pm 0.01$ & $2.34 \pm 0.40$ \\
\hline 1500 & $1.75 \pm 0.01$ & $1.77 \pm 0.01$ & $1.94 \pm 0.01$ & $2.22 \pm 0.13$ & $1.49 \pm 0.02$ & $1.66 \pm 0.01$ & $1.71 \pm 0.03$ & $1.69 \pm 0.01$ & $3.87 \pm 1.00$ \\
\hline 1250 & $1.75 \pm 0.01$ & $1.77 \pm 0.01$ & $1.94 \pm 0.01$ & $2.25 \pm 0.13$ & $1.50 \pm 0.03$ & $1.67 \pm 0.01$ & $1.74 \pm 0.04$ & $1.69 \pm 0.02$ & $5.20 \pm 1.07$ \\
\hline 1000 & $1.75 \pm 0.01$ & $1.77 \pm 0.01$ & $1.94 \pm 0.01$ & $2.29 \pm 0.15$ & $1.53 \pm 0.03$ & $1.68 \pm 0.01$ & $1.78 \pm 0.04$ & $1.69 \pm 0.02$ & $6.56 \pm 1.41$ \\
\hline 750 & $1.75 \pm 0.02$ & $1.77 \pm 0.02$ & $1.94 \pm 0.01$ & $2.32 \pm 0.18$ & $1.55 \pm 0.04$ & $1.69 \pm 0.02$ & $1.83 \pm 0.05$ & $1.69 \pm 0.02$ & $6.95 \pm 1.29$ \\
\hline
\end{tabular}


Table S24. Spearman correlation coefficients produced by all models on the CASF-2016 test set

\begin{tabular}{|c|c|c|c|c|c|c|c|c|c|}
\hline $\begin{array}{l}\text { Training } \\
\text { set size }\end{array}$ & X-Score ${ }^{H M}$ & ChemScore & ASP & L-SVR & RF & KNN & DT & BRR & MLP \\
\hline 3000 & $0.617 \pm 0.002$ & $0.604 \pm 0.003$ & $0.570 \pm 0.005$ & $0.686 \pm 0.005$ & $0.790 \pm 0.005$ & $0.699 \pm 0.003$ & $0.674 \pm 0.011$ & $0.693 \pm 0.001$ & $0.704 \pm 0.006$ \\
\hline 2750 & $0.618 \pm 0.003$ & $0.603 \pm 0.003$ & $0.570 \pm 0.006$ & $0.683 \pm 0.008$ & $0.783 \pm 0.007$ & $0.695 \pm 0.003$ & $0.666 \pm 0.012$ & $0.692 \pm 0.002$ & $0.701 \pm 0.007$ \\
\hline 2500 & $0.617 \pm 0.002$ & $0.604 \pm 0.003$ & $0.570 \pm 0.007$ & $0.682 \pm 0.010$ & $0.779 \pm 0.007$ & $0.690 \pm 0.004$ & $0.660 \pm 0.014$ & $0.691 \pm 0.003$ & $0.692 \pm 0.015$ \\
\hline 2250 & $0.617 \pm 0.003$ & $0.602 \pm 0.005$ & $0.568 \pm 0.007$ & $0.679 \pm 0.012$ & $0.773 \pm 0.009$ & $0.685 \pm 0.005$ & $0.655 \pm 0.014$ & $0.692 \pm 0.003$ & $0.680 \pm 0.029$ \\
\hline 2000 & $0.618 \pm 0.004$ & $0.604 \pm 0.005$ & $0.571 \pm 0.009$ & $0.675 \pm 0.016$ & $0.765 \pm 0.010$ & $0.682 \pm 0.006$ & $0.643 \pm 0.016$ & $0.690 \pm 0.004$ & $0.671 \pm 0.040$ \\
\hline 1750 & $0.617 \pm 0.005$ & $0.603 \pm 0.006$ & $0.569 \pm 0.010$ & $0.670 \pm 0.019$ & $0.757 \pm 0.013$ & $0.678 \pm 0.005$ & $0.631 \pm 0.019$ & $0.690 \pm 0.005$ & $0.668 \pm 0.029$ \\
\hline 1500 & $0.617 \pm 0.005$ & $0.602 \pm 0.006$ & $0.570 \pm 0.013$ & $0.666 \pm 0.022$ & $0.748 \pm 0.012$ & $0.676 \pm 0.005$ & $0.620 \pm 0.017$ & $0.690 \pm 0.006$ & $0.539 \pm 0.096$ \\
\hline 1250 & $0.617 \pm 0.006$ & $0.601 \pm 0.007$ & $0.569 \pm 0.012$ & $0.655 \pm 0.031$ & $0.740 \pm 0.013$ & $0.675 \pm 0.006$ & $0.607 \pm 0.023$ & $0.689 \pm 0.007$ & $0.442 \pm 0.101$ \\
\hline 1000 & $0.617 \pm 0.007$ & $0.602 \pm 0.007$ & $0.569 \pm 0.015$ & $0.649 \pm 0.035$ & $0.728 \pm 0.017$ & $0.676 \pm 0.007$ & $0.586 \pm 0.021$ & $0.688 \pm 0.007$ & $0.365 \pm 0.116$ \\
\hline 750 & $0.614 \pm 0.009$ & $0.599 \pm 0.009$ & $0.563 \pm 0.019$ & $0.641 \pm 0.042$ & $0.714 \pm 0.019$ & $0.673 \pm 0.008$ & $0.558 \pm 0.028$ & $0.685 \pm 0.011$ & $0.318 \pm 0.127$ \\
\hline
\end{tabular}




\section{PART 9. List of the non-redundant, standard training sets compiled based on the PDBbind refined set}

The non-redundant, standard training sets compiled based on the PDBbind refined set are given in separate index files (in plain TXT format) as follows. Each index file lists the PDB code of the complex structure, resolution of the crystal structure, release year, binding affinity data, and the code of the ligand molecule as in PDB. The processed structures of all relevant complexes are included in the corresponding release of the PDBbind refined set, which is available from the PDBbind-CN database at http:/ / www.pdbbind-cn.org/.

\begin{tabular}{|c|c|}
\hline Name of the index file & Annotation \\
\hline INDEX_NR_dataset_0.80_2016.txt & $\begin{array}{l}\text { Training set selected from the refined set v. } 2016 \text { by a } \\
\text { similarity threshold of } 80 \%\end{array}$ \\
\hline INDEX_NR_dataset_0.85_2016.txt & $\begin{array}{l}\text { Training set selected from the refined set v. } 2016 \text { by a } \\
\text { similarity threshold of } 85 \%\end{array}$ \\
\hline INDEX_NR_dataset_0.90_2016.txt & $\begin{array}{l}\text { Training set selected from the refined set v.2016 by a } \\
\text { similarity threshold of } 90 \%\end{array}$ \\
\hline INDEX_NR_dataset_0.95_2016.txt & $\begin{array}{l}\text { Training set selected from the refined set v. } 2016 \text { by a } \\
\text { similarity threshold of } 95 \%\end{array}$ \\
\hline INDEX_NR_dataset_0.80_2017.txt & $\begin{array}{l}\text { Training set selected from the refined set v. } 2017 \text { by a } \\
\text { similarity threshold of } 80 \%\end{array}$ \\
\hline INDEX_NR_dataset_0.85_2017.txt & $\begin{array}{l}\text { Training set selected from the refined set v. } 2017 \text { by a } \\
\text { similarity threshold of } 85 \%\end{array}$ \\
\hline INDEX_NR_dataset_0.90_2017.txt & $\begin{array}{l}\text { Training set selected from the refined set v. } 2017 \text { by a } \\
\text { similarity threshold of } 90 \%\end{array}$ \\
\hline INDEX_NR_dataset_0.95_2017.txt & $\begin{array}{l}\text { Training set selected from the refined set v. } 2017 \text { by a } \\
\text { similarity threshold of } 95 \%\end{array}$ \\
\hline INDEX_NR_dataset_0.80_2018.txt & $\begin{array}{l}\text { Training set selected from the refined set v. } 2018 \text { by a } \\
\text { similarity threshold of } 80 \%\end{array}$ \\
\hline INDEX_NR_dataset_0.85_2018.txt & $\begin{array}{l}\text { Training set selected from the refined set v. } 2018 \text { by a } \\
\text { similarity threshold of } 85 \%\end{array}$ \\
\hline INDEX_NR_dataset_0.90_2018.txt & $\begin{array}{l}\text { Training set selected from the refined set v. } 2018 \text { by a } \\
\text { similarity threshold of } 90 \%\end{array}$ \\
\hline INDEX_NR_dataset_0.95_2018.txt & $\begin{array}{l}\text { Training set selected from the refined set v. } 2018 \text { by a } \\
\text { similarity threshold of } 95 \%\end{array}$ \\
\hline
\end{tabular}


PART 10. Performance of all scoring functions calibrated on the non-redundant training sets selected from several versions of the PDBbind refined set

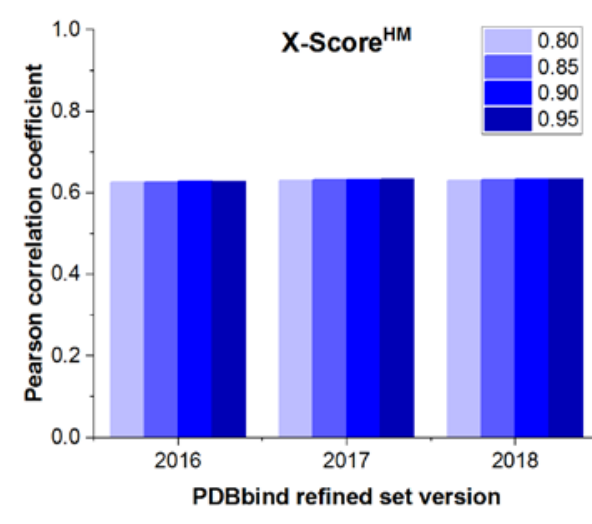

(A)

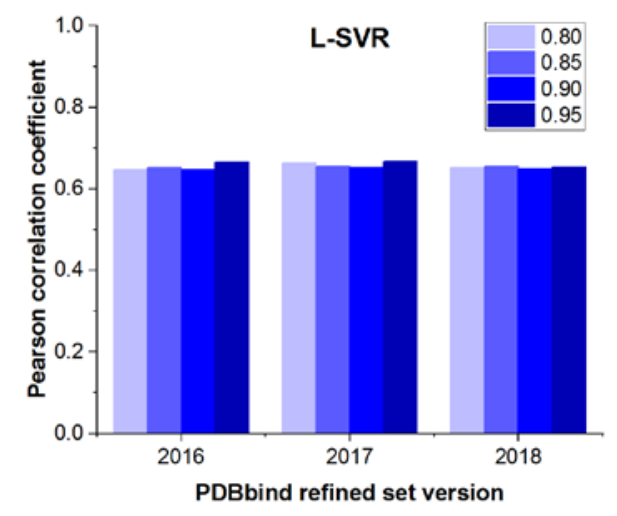

(D)

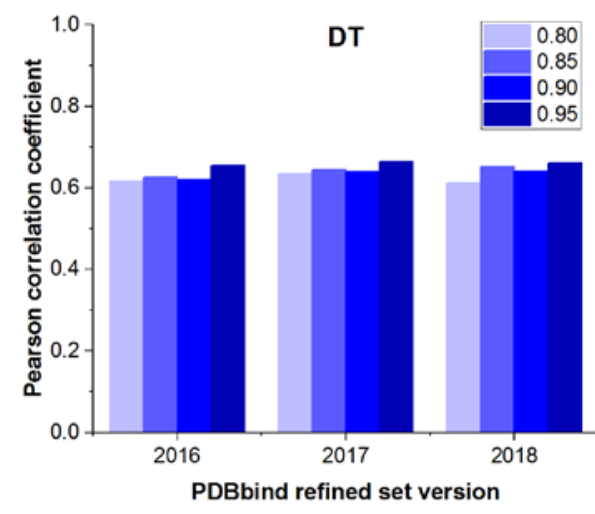

(G)

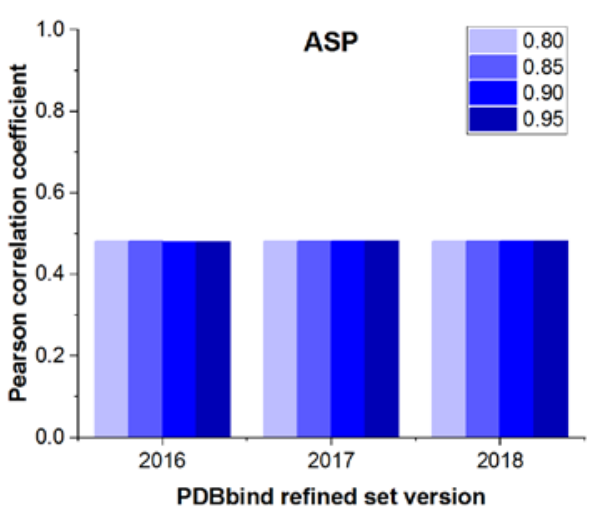

(B)

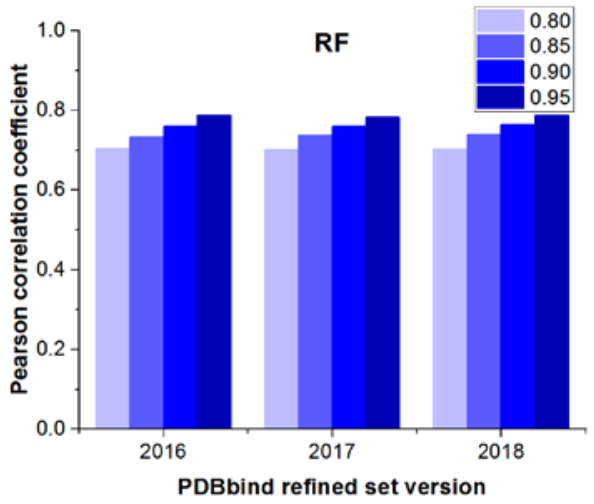

(E)

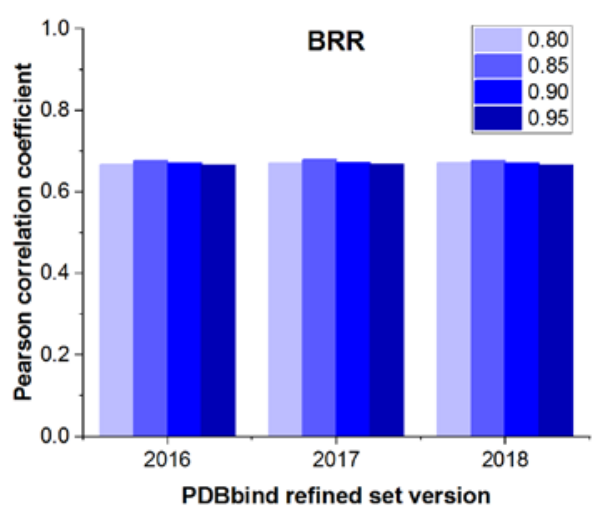

(H)

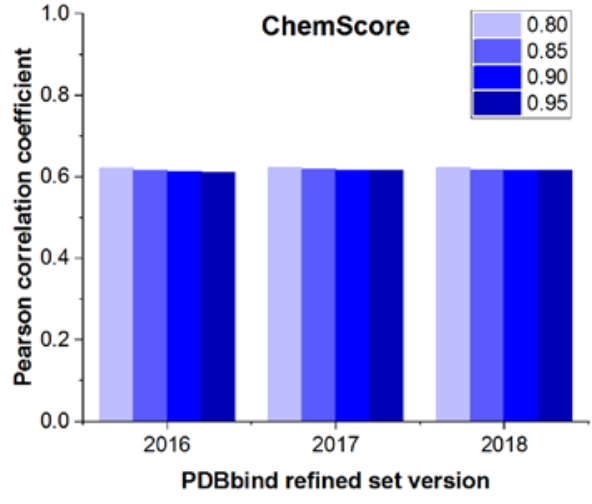

(C)

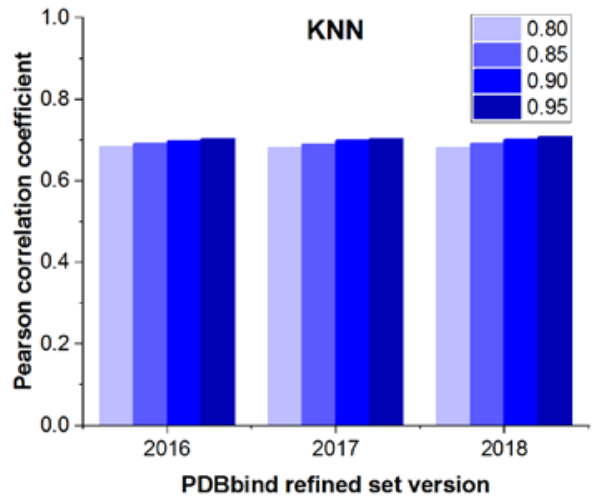

(F)

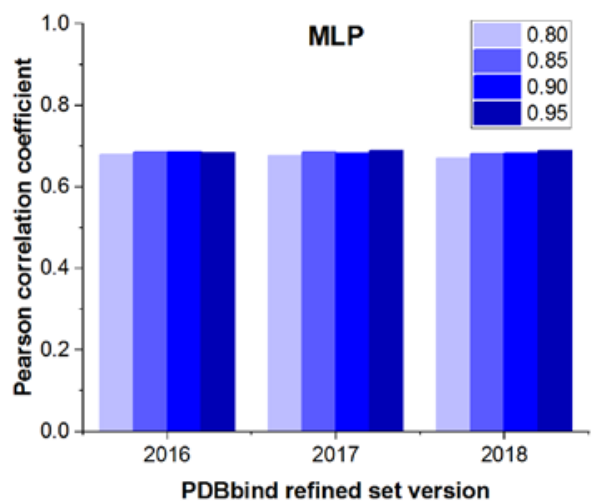

(I)

Figure S1. Performance of all scoring functions in non-redundant training sets originated from different versions of Refined set in PDBbind database. Different colors represent the similarity cutoff of the nonredundant training sets. 